\title{
A PSF-based Approach to TESS High quality data Of Stellar clusters (PATHOS) - II. Search for exoplanets in open clusters of the southern ecliptic hemisphere and their frequency.
}

\author{
D. Nardiello ${ }^{1,2 \star}$, G. Piotto ${ }^{2,3}$, M. Deleuil ${ }^{1}$, L. Malavolta $^{3}$, M. Montalto ${ }^{2,3}$, \\ L. R. Bedin ${ }^{2}$, L. Borsato 2 , V. Granata ${ }^{2,3}$, M. Libralato ${ }^{4}$, E. E. Manthopoulou ${ }^{2,3}$ \\ ${ }^{1}$ Aix Marseille Univ, CNRS, CNES, LAM, Marseille, France \\ ${ }^{2}$ Istituto Nazionale di Astrofisica - Osservatorio Astronomico di Padova, Vicolo dell'Osservatorio 5, IT-35122, Padova, Italy \\ ${ }^{3}$ Dipartimento di Fisica e Astronomia "Galileo Galilei", Università di Padova, Vicolo dell'Osservatorio 3, IT-35122, Padova, Italy \\ ${ }^{4}$ Space Telescope Science Institute, 3800 San Martin Drive, Baltimore, MD 21218, USA
}

Accepted 2020 May 20. Received 2020 May 20; in original form 2020 April 22.

\begin{abstract}
The scope of the project "A PSF-based Approach to TESS High Quality data Of Stellar clusters" (PATHOS) is the extraction and analysis of high-precision light curves of stars in stellar clusters and young associations for the identification of candidate exoplanets and variable stars. The cutting-edge tools used in this project allow us to measure the real flux of stars in dense fields, minimising the effects due to contamination by neighbour sources. We extracted about 200000 light curves of stars in 645 open clusters located in the southern ecliptic hemisphere and observed by TESS during the first year of its mission. We searched for transiting signals and we found 33 objects of interest, 11 of them are strong candidate exoplanets. Because of the limited S/N, we did not find any Earth or super-Earth. We identified two Neptune-size planets orbiting stars with $R_{\star}<1.5 R_{\odot}$, implying a frequency $f_{\star}=1.34 \pm 0.95 \%$, consistent with the frequency around field stars. The 7 Jupiter candidates around stars with $R_{\star}<1.5 R_{\odot}$ imply a frequency $f_{\star}=0.19 \pm 0.07 \%$, smaller than in the field. A more complete estimate of the survey completeness and false positive rate is needed to confirm these results. Light curves used in this work will be made available to the astronomical community on the Mikulski Archive for Space Telescope under the project PATHOS.
\end{abstract}

Key words: techniques: image processing - techniques: photometric - stars: planetary systems - Galaxy: open clusters and associations: general

\section{INTRODUCTION}

In our Galaxy there are more than 1500 among globular clusters, open clusters and stellar associations. These objects can comprise up to several hundreds thousand stars covering all ranges of spectral classes and stellar evolutionary stages and have ages that span from few million years to almost the age of the Universe. Their chemical properties reflect the chemical evolution of the Milky Way. For these stars we can extract with high accuracy essential pieces of information, like the radius, the mass, the chemical content, and the age, i.e. parameters that suffer usually of large uncertainties for many field stars. The possibility of measuring

^ E-mail: domenico.nardiello@lam.fr stellar host parameters with high precision makes the search for exoplanets around cluster stars, and their frequency, of particular interest, especially if we want to comprehend how they formed and evolved in different environments.

Despite the large number of stars in stellar clusters and associations, only a small fraction of known (candidate) exoplanets orbits these stars. These objects have been found by looking for transits in the light curves of the cluster/association members and/or analysing their radial velocities. To date, 28 between confirmed and candidate exoplanets have been discovered in stellar clusters and associations. The first exoplanet discovered in a stellar cluster, the Hyades, was found by Sato et al. (2007); since then, other three exoplanets and an exoplanetary system have been found in this open cluster, both by using radial velocities 
(Quinn et al. 2014) and Kepler/K2 (Howell et al. 2014) light curves (David et al. 2016a; Mann et al. 2016b; Ciardi et al. 2018; Mann et al. 2018; Vanderburg et al. 2018) The first two transiting exoplanets in an open cluster, NGC 6811, were discovered by Meibom et al. (2013) using Kepler main mission data (Borucki et al. 2010). The Praesepe cluster hosts 8 candidate/confirmed exoplanets and an exoplanetary system (Quinn et al. 2012; Barros et al. 2016; Libralato et al. 2016b; Malavolta et al. 2016; Obermeier et al. 2016; Pope et al. 2016; Mann et al. 2017; Pepper et al. 2017; Gaidos et al. 2020). A transiting exoplanet has been discovered by Curtis et al. (2018) in the 3 Gyr old open cluster Ruprecht 147. No transiting exoplanets around members of the old open cluster M 67 have been reported by Nardiello et al. (2016b), despite Brucalassi et al. (2014, 2016, 2017) found an excess of hot Jupiters by using radial velocity measurements. Noteworthy, the unusual null detection of exoplanets around Pleiades members has been reported by Gaidos et al. (2017) and by Mann et al. (2017). Some exoplanets have also been found in young associations and moving groups. The first one is $\mathrm{K} 2-33 \mathrm{~b}$, orbiting a pre-main sequence star that belongs to the Upper Scorpius OB association (David et al. 2016b; Mann et al. 2016a). Exoplanets were also found in the associations Cas-Tau (EPIC 247267267 b, David et al. 2018) and Tuc-Hor (DS Tuc A b, Newton et al. 2019; Benatti et al. 2019).

Among the previously mentioned exoplanets in stellar clusters and associations, 18 are transiting exoplanets and, excluding DS Tuc A b, they were all discovered by using data collected with Kepler. But only a handful of clusters and associations were observed during the Kepler main and K2 missions, and the number of studied cluster members is limited to few thousand stars. The opportunity that the Transiting Exoplanet Survey Satellite (TESS, Ricker et al. 2015) is giving us is without equal: at the end of its 2-years nominal mission, this satellite will have observed more than $80 \%$ of the sky, thus including almost all the known clusters and associations.

In TESS observations, the large part of the cluster members fall in 30-minute cadence stacked frames, called Full Frame Images (FFIs), collected during 27-days campaigns; each campaign covers a sector of the sky of $24 \times 96 \mathrm{deg}^{2}$. One of the major problems to be addressed in the extraction of high precision light curves of cluster members from TESS FFIs is related to their low angular resolution $\left(\sim 21 \operatorname{arcsec} \mathrm{pix}^{-1}\right)$. In fact, even low density stellar clusters appear crowded in TESS images, where their members are likely blended among each others or with field stars. In these conditions, the extraction of light curves using simple aperture photometry is not reliable, because contamination and blending effects prevail.

In the last years, many approaches have been developed for the extraction of high precision light curves of stars in crowded regions, based on the use of the Point Spread Functions (PSFs, Nardiello et al. 2015a; Libralato et al. 2016a) or the Difference Imaging Analysis (Alard \& Lupton 1998; Chang et al. 2015; Soares-Furtado et al. 2017; Bouma et al. 2019; Wallace et al. 2019; Soares-Furtado et al. 2020). In the first paper of this series we presented our project "A PSF-based Approach to TESS High Quality data Of Stellar clusters" (PATHOS, Nardiello et al. 2019, hereafter Paper I), aimed at the extraction of high precision light curves for stars in stellar clusters (open and globular clusters) and young associations. Our approach of light curve extraction, already widely tested on ground-based, Kepler and also TESS data, is based on the use of empirical PSFs and an input catalogue to measure the flux of a given star after having suppressed the contribution of its neighbours. In this way we are able to measure the real flux of the target star, minimise the dilution effects due to the contamination by neighbour sources (essential in the search for exoplanetary transits), and extract high precision photometry even for stars close to the TESS limiting magnitude $(T \sim 17)$.

In Paper I we successfully applied our method to an extreme case of crowded region, a field containing the globular cluster 47 Tuc, which includes Galactic and Small Magellanic Cloud stars. We searched for exoplanets and variable stars among all the extracted light curves, finding one candidate exoplanet (a hot Jupiter around a field star, PATHOS-1) and many new variable stars along the red and asymptotic giant branch sequences of 47 Tuc. For this work, we applied our PSF-based approach to extract high-precision light curves for stars belonging to open clusters in the southern ecliptic hemisphere and listed in the catalogue published by CantatGaudin et al. (2018, Sect. 2). We analysed these light curves searching for transit signals, we selected the most promising transiting objects and validated them with a series of vetting tests (Sect. 3). We modelled the transits of the candidate exoplanets to derive their parameters (Sect. 4), and analysed their dependence on cluster properties (Sects. 4 and 5). We also estimated the observed and expected occurrence rate of exoplanets and discussed our results in Section 6 .

\section{OBSERVATIONS AND DATA REDUCTION}

This work is focused on the open clusters located in the southern ecliptic hemisphere and that were observed by TESS in Sectors 1-13. In this work we used observations carried out by TESS in a period of $\sim 1$ year (357 days), between 2018 July 25 and 2019 July 17; the total number of FFIs used for the extraction of the light curves is 245576 , that are all the downloadable FFIs for the Sectors 1-13 ${ }^{1}$.

The light curve extraction has been performed using an improved version of img2lc, the software described in Paper I. The three main ingredients of our approach are: (i) Full Frame Images, (ii) PSFs, and (iii) input catalogue. In the following, we give a short description of the input catalogue adopted and of our pipeline.

\subsection{The input catalogue}

Our pipeline extracts the light curves of all the sources which coordinates are listed in an input catalogue. For this work we used as input the catalogue published by Cantat-Gaudin et al. (2018). This catalogue contains, for 1229 clusters, a list of stellar cluster members, whose membership probabilities are calculated by using Gaia DR2 proper motions and parallaxes (Gaia Collaboration et al. 2018). From this

1 https://archive.stsci.edu/tess/bulk_downloads/bulk_downloads_ffi-tp-lc-dv.html 


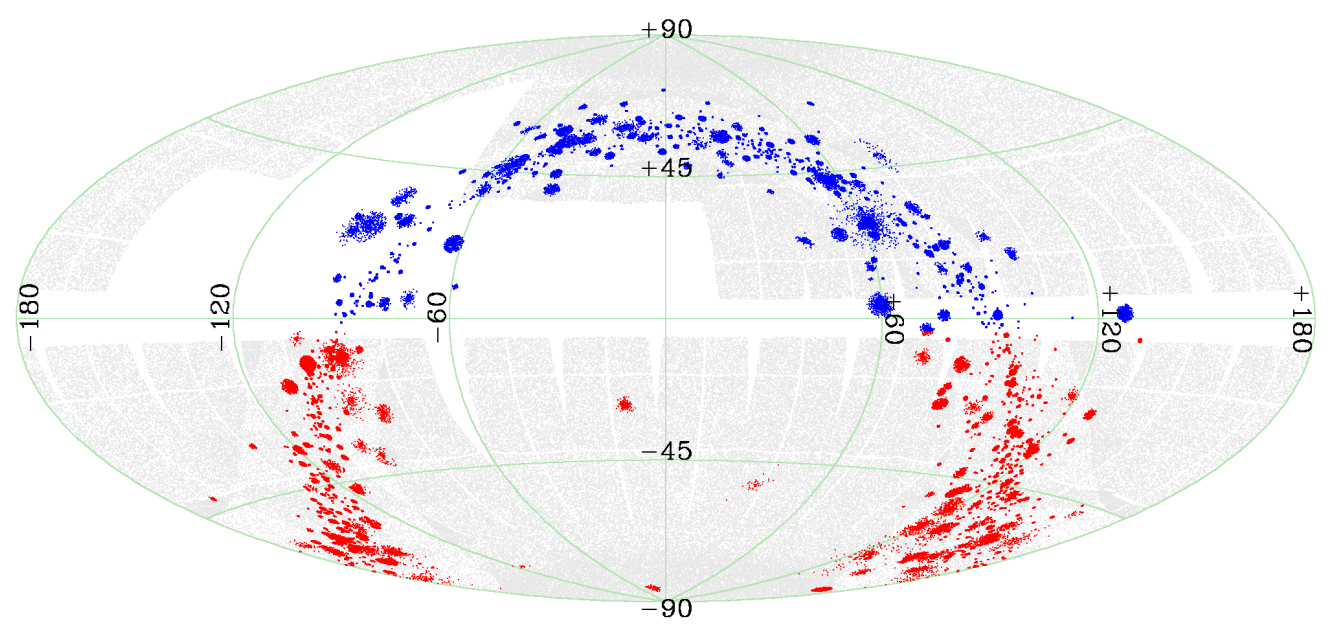

Figure 1. Aitoff projection in ecliptic coordinates of the TESS observations: grey points represent the sources observed in 2-min cadence mode in Sectors 1-24; red/blue points are the open cluster members for which we propose to extract the light curves in our project; red points are the stars in the input catalogue used in this work.

catalogue we selected a sub-sample of stars having magnitude $G<17.5$ and (a conservative) ecliptic latitude $\beta<-4^{\circ}$, that corresponds to the fields covered by TESS in the Sectors 1-13. In this way we selected a total of 189090 cluster members, although $\sim 13.9 \%$ of them are not observed by TESS, because they fall between CCD/Camera gaps or between sector gaps, or because they are outside the field of view of TESS observations, as shown in Figure 1. On the other hand, $\sim 1 / 4$ of the stars in the input catalogue are observed in more than one sector. Finally, we extracted a total of 219256 light curves of stars in 645 open clusters. In the following analysis we excluded the members of NGC 1901, an open cluster in the TESS continuous viewing zone; this cluster is the only one observed in all the 13 Sectors, and will be the subject of a future work (Manthopoulou et al., in preparation).

\subsection{Light curve extraction}

For the extraction of the light curves from TESS FFIs, we adopted the PSF-based approach developed by Nardiello et al. (2015a, 2016a) for the ground-based data collected with the Asiago Schmidt Telescope $67 / 92 \mathrm{~cm}$. This pipeline was also adapted to Kepler/K2 space-based data-set by Libralato et al. (2016a, see also Libralato et al. 2016b; Nardiello et al. 2016b) and finally to TESS data in Paper I.

As a first step, the routine transforms the positions and the luminosities of the stars in the input catalogue into the reference system of a single image. For all the stars in the input catalogue, it transforms the $(\alpha, \delta)$-coordinates into the image reference system using the transformation coefficients listed in the FITS header of the single FFI.

In a second step, the software calculates the photometric zero-point between the calibrated and instrumental TESS magnitudes as follows: for the 200 brightest, not saturated stars of each FFI, it extracts the PSF-fitting magnitudes $T_{\text {inst }}$, and calculates the 3.5- $\sigma$-clipped mean value of $T_{\text {cal }}^{i}-T_{\text {inst }}^{i}$, with $i=1, \ldots, 200$ and $T_{\text {cal }}$ the calibrated TESS magnitudes calculated by using Gaia DR2 magnitudes and the equation in Stassun et al. (2019).

Finally, for each star in the input catalogue, the routine searches in the Gaia DR2 catalogue (Gaia Collaboration et al. 2018) all the neighbours located between 0.5 and 20 TESS pixels from the target star and having $T<17.5$, and transforms their positions and luminosities in the FFI reference system, as described above. The software models the neighbour sources by using the local PSF and the transformed positions and luminosities, and it subtracts these models to the original FFI, as shown in Fig. 2. After the neighbour-subtraction, our software performs PSF-fitting and four different aperture photometries (1-, 2-, 3-, and 4pixels radius) of the target star.

\subsection{Systematic effects correction}

Variations of the spacecraft, detector and environment conditions affect the quality of the light curves, introducing systematic artifacts. These systematic trends are common to all the stars of a given TESS Camera/CCD/Sector, and can be corrected using orthonormal functions called cotrending basis vectors (CBVs).

To extract the CBVs, we combined two samples of light curves: (i) the light curves of the stars in the input catalogue described in Sect. 2.1 and (ii) the light curves of the stars also observed in 2-minute cadence mode (grey points of Fig. 1) and extracted from FFIs with our pipeline. We extracted the second sample of light curves to increase the number of bright stars in our list and extract better CBVs associated to 3- and 4-pixel aperture photometries.

To extract the CBVs, we followed a procedure similar to that described in Paper I: for each star and for each photometric method, we calculated the raw $\mathrm{RMS}^{2}$. On the basis of the RMS distributions, we identified the magnitude interval where each photometric method works better and we

\footnotetext{
2 Defined as the 68.27th-percentile of the sorted residual from the median value of the light curve
} 

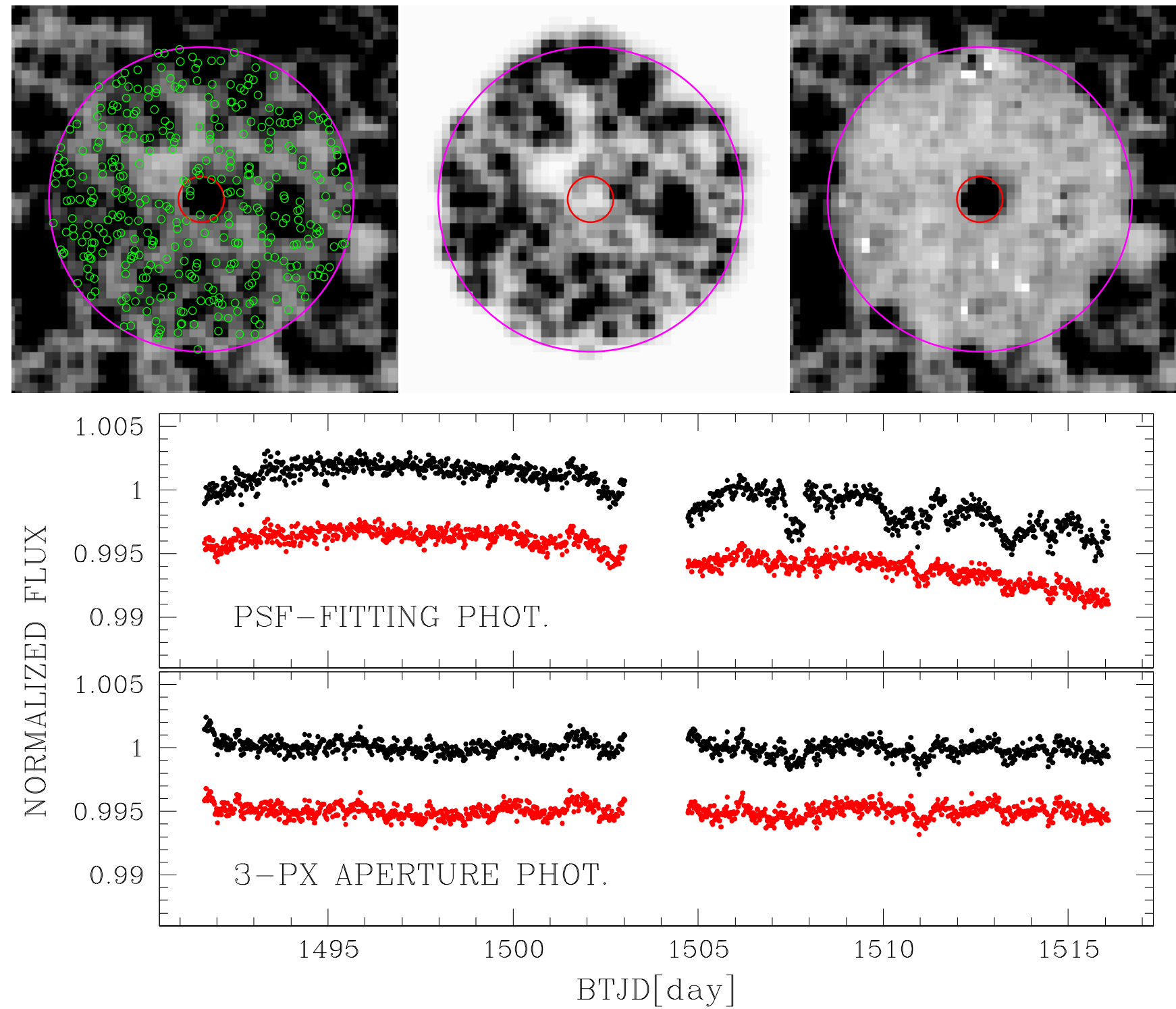

Figure 2. From FFI to the final light curve. Top Panels. Example of neighbour subtraction. Left-hand panel shows the original FFI (tess2019009222936-s0007-1-1-0131-s_ffic.fits) centred on the star Gaia DR2 3111381928222301184, a possible cluster member of Gulliver 47; red circle is the photometric aperture used to analyse this star, green circles are the neighbours located within a radius of 20 pixels (magenta circle) from the target star. Middle panel shows the models of the neighbours subtracted from the FFI; right-hand panel is the FFI after neighbour subtraction. Bottom Panels. The PSF-fitting and 3-pixel aperture photometry light curves of the star Gaia DR2 3111381928222301184 before (black points) and after (red points) the systematic correction.
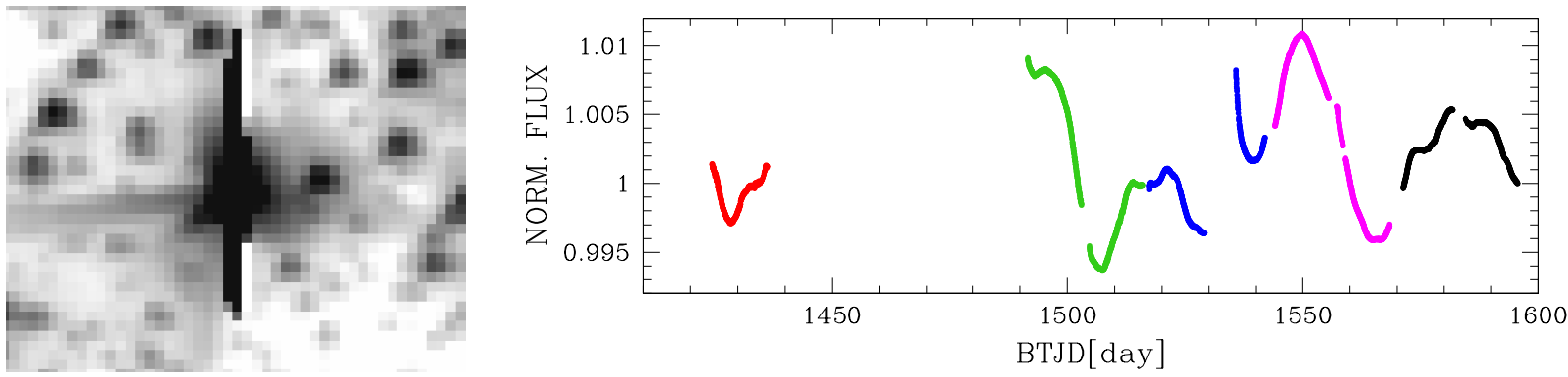

Figure 3. Left-hand panel shows the saturated star Gaia DR2 05290818758516639104 on the FFI tess2019010175936-s0007-4-1-0131s_ffic.fits. Right-hand panel is its light curve extracted from Sectors 4 (red), 7 (green), 8 (blue), 9 (magenta), and 10 (black). 
selected the best measured stars on the basis of their RMS values as follows: for each photometric method, we divided the RMS distributions in bins of width $0.75 \mathrm{~T}$ magnitude and we estimated in each magnitude interval the $3.5 \sigma$-clipped mean value of the RMS. We interpolated the binned points with a spline: for each photometric method, we selected the magnitude interval where the mean RMS distribution is lower compared to the other distributions. For the stars that passed the first selection criteria, we calculated the median of the correlation coefficients between a given light curve and the light curves of the other stars. We kept all the stars with a median correlation coefficient $>50 \%$, and we iterated other 2 times, restarting from the calculation of the median correlation coefficient, and considering at each iteration only the stars that passed the selection criteria of the previous iteration. With the surviving stars, we extracted $20 \mathrm{CBVs}$ for each photometric method using the single value decomposition. On average, CBVs are obtained by using between 200 and 300 stars.

To correct the light curves, we used the LevenbergMarquardt method (Moré et al. 1980) to find the coefficients $A_{i}$ that minimised the expression:

$F_{\text {raw }}^{j}-\sum_{i}\left(A_{i} \cdot \mathrm{CBV}_{i}^{j}\right)$

with $F_{\text {raw }}^{j}$ the raw flux of the light curve at the epoch $j$, and $\mathrm{CBV}_{i}$ the $i$-th $\mathrm{CBV}$, with $i=1, \ldots, 20$. We changed the number of CBVs applied to obtain the correction from 1 to 20 , and, for each light curve and photometric method, we selected the number of CBVs whose correction gives the lower Akaike's information criterion (AIC) score.

Bottom panels of Fig. 2 show the light curves of Gaia DR2 3111381928222301184 before (black points) and after (red points) correction, in the case of PSF-fitting photometry and 3-pixel aperture photometry: the improvement of the light curve in the case of PSF-fitting photometry is clear, and the RMS of the light curve passes from $2.1 \mathrm{mmag}$ to 1.6 mmag.

\subsection{Saturated stars}

Stars with $T \lesssim 5.5$ are saturated on FFIs, and form a bleeding column. As explained in the TESS Instrument Handbook, the CCDs conserve the electrons even when saturation causes their moving along the columns neighbouring the pixel where they were generated. To recover the charge along the bleeding column we used the technique described in Gilliland (2004) and Gilliland et al. (2010), and already tested in many works based on Hubble Space Telescope data (see, e.g., Anderson et al. 2008; Bellini et al. 2017; Nardiello et al. 2018). Briefly, our routine checks if the central pixel and three of the neighbouring pixels have counts $>80000 e^{-} / s$; in this case, it checks the presence of a bleeding column and retraces its shape. Finally, it adds the contribution that comes from the bleeding columns to the flux calculated inside an aperture of radius 5.5 pixels centred on the star. In the case of saturated stars, the routine did not perform neighbour subtraction and we did not correct for systematic trends, because the sample of saturated (and not variable) stars is too small to extract CBVs. Figure 3 shows the saturated star Gaia DR2 05290818758516639104 $(T \sim 3.1)$ on a FFI (left panel) and its light curve extracted from 5 sectors (right panel). The standard deviation from the mean value of the light curve, after removing the contribution of the variability, is $\sim 26 \mathrm{ppm}$.

\subsection{Data release}

All the light curves extracted in this work are released on the Mikulski Archive for Space Telescopes (MAST) as a High Level Science Product (HLSP) under the project PATHOS ${ }^{3}$ (DOI: 10.17909/t9-es7m-vw14). Each light curve (in fits and ascii format) contains the epoch in TESS Barycentric Julian Day (BTJD), the five extracted raw and corrected photometries (PSF-fitting, 1-pixel, 2-pixel, 3-pixel, 4-pixel aperture), the value of the local sky, the position ( $\mathrm{x}, \mathrm{y}$ ) on the image, and the data quality flag DQUALITY (see TESS Science Data Products Description Document for details). All the main information on the star extracted from the Gaia DR2 catalogue (Gaia Collaboration et al. 2018) and on the observations are reported in the header of each light curve.

\subsection{Photometric precision}

Figure 4 shows the two quality parameters adopted also in Paper I and previous works of our group: (i) the RMS, calculated using the cotrended light curves and defined as the 68.27 th-percentile of the sorted residual from the median value, obtained clipping-out the outliers in 10 iterations; (ii) the P2P RMS (point-to-point), that is not sensitive to intrinsic stellar variability, and obtained calculating the 68.27 th percentile of the distribution of the sorted residual from the median value of $\delta F_{j}=F_{j}-F_{j+1}$, with $F_{j}$ and $F_{j+1}$ the flux of the light curve at the epochs $j$ and $j+1$.

For each photometric method, we derived, for all the extracted light curves, the mean trends of the RMS and P2P RMS as a function of the $T$ magnitude as follows: we divided each parameter distribution in intervals of $0.75 \mathrm{~T}$ magnitude and we calculated in each bin the $3.5 \sigma$-clipped average of the parameter. Finally, we interpolated the mean values with a cubic spline. Figure 4 shows these mean trends both for the RMS (top panel) and for the P2P RMS (bottom panel); in the plot, different colours are associated to different photometric methods: black, magenta, blue, green, and yellow lines correspond to PSF-fitting, 1-, 2-, 3-, and 4-pixel aperture photometries, respectively. Red stars are the saturated stars, measured as described in Sect. 2.4.

We used the P2P RMS mean trends to select the best photometric method for each light curve: given a star of magnitude $T_{\star}$, we have chosen to analyse the light curve extracted with the photometric method that in $T_{\star}$ have the lower mean P2P RMS. On average, for not saturated stars with $T \lesssim 7.0$, we used the 4-pixel aperture photometry; for stars with $7.0 \lesssim T \lesssim 9.0$ and with $9.0 \lesssim T \lesssim 10.5$ the best photometric methods are the 3-pixel and 2-pixel aperture photometries, respectively. PSF-fitting photometry works well for stars having $10.5 \lesssim T \lesssim 13.5$; finally, in the faint regime of magnitudes $(T \gtrsim 13.5)$ the 1-pixel aperture photometry gives the best results. Gray dashed line in Fig. 4 is the theoretical limit obtained taking into account all the sources

\footnotetext{
3 https://archive.stsci.edu/hlsp/pathos
} 

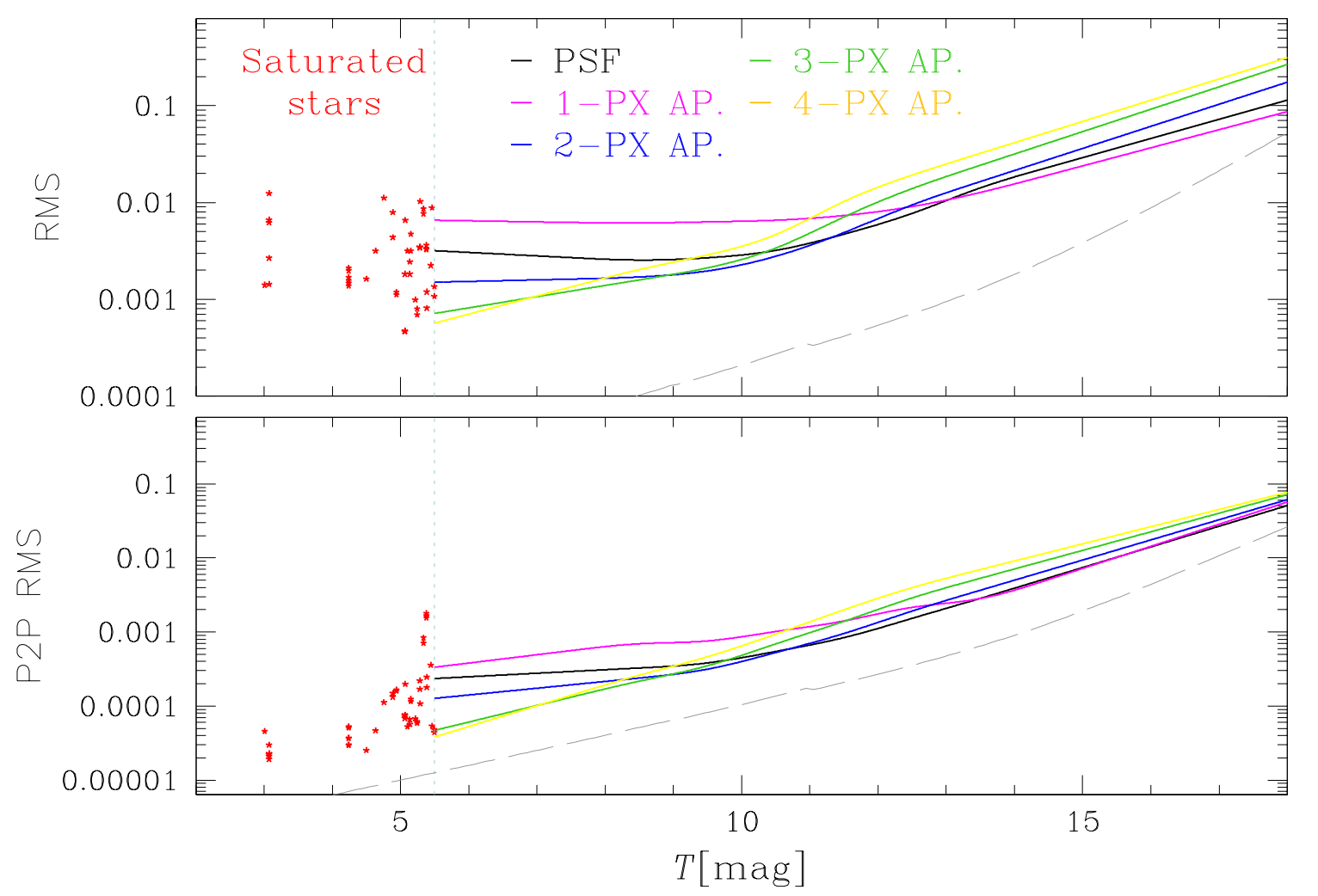

Figure 4. Photometric RMS (top panel) and P2P RMS (bottom panel) mean trends as a function of the TESS magnitude for all the light curves extracted in this work, colour-coded on the basis of the photometric method adopted: black for PSF-fitting photometry, magenta, blue, green, and yellow for 1-pixel, 2-pixel, 3-pixel, and 4-pixel aperture photometries, respectively. Red points represent the saturated stars. The grey dashed lines represent the theoretical limit.

of noise (shot noise, sky, readout-noise RON, and dark current $\mathrm{DC}$ ), adopting the average values of $\mathrm{RON} \sim 9 e^{-} \mathrm{px}^{-1}$, $\mathrm{DC} \sim 1 e^{-} s^{-1} \mathrm{px}^{-1}$, sky $\sim 150 e^{-} s^{-1}$, and using in different intervals of magnitudes the aperture radius where the light curve shows the lower scatter, on the basis of the considerations done previously. Theoretical limit distributions are, as expected, lower then the mean observed trends; this is mainly due to a combination of different effects, like stellar variability (that mainly affects the RMS distributions), variations of the background in the light curves, contamination by and blending with not-subtracted sources, etc.

Before of the analysis, we excluded all the light curves for which instrumental magnitude $T_{\text {instr }}$ is too different from the expected calibrated magnitude $T_{\text {calib }}$, by using the following procedure: for each photometric method (and for each Sector), we analysed the $\delta T=T_{\text {instr }}-T_{\text {calib }}$ distribution, and we excluded all the light curves whose $\delta T$ value deviates more than $4 \sigma$ from the mean value. In this way we excluded all the light curves of stars strongly contaminated by other sources that were not subtracted during the light curve extraction (e.g., close bleeding columns, hot pixels, background galaxies or other sources that were not in the Gaia DR2 catalogue). We also excluded all the light curves that have $<75 \%$ of good points (i.e. DQUALITY $=0$ and FLUX $\neq 0$ ). Finally, we analysed 196231 light curves associated to 147702 stars.

\section{CANDIDATE EXOPLANETS SELECTION}

In order to select candidate transiting exoplanets orbiting stellar cluster members, we performed a series of analyses and tests on the light curves selected as described in the previous sections, to identify and remove the large part of false positive events.

As a first step, we removed the variability of the stars. We flattened the light curves using 5 th order splines defined on $N_{\text {knots }}$ knots. We calculated two different grid of knots: (i) a knot every 6.5-hours, and (ii) a knot every 13hours. In this way, we were able to better model short- and long-period variable stars, but also to prevent the flattening of the transits whose duration is longer than 6.5 hours. In this phase we also "cleaned" each light curve, excluding: (i) points whose quality flag was DQUALITY>0; (ii) all the outliers above $3.5 \sigma$ the median flux; (iii) the photometric points associated to local sky background higher than $5 \sigma_{\text {sky }}$ above median value of the sky. Panels (a) and (b) of Fig. 5 show the procedure of flattening of the light curve of the star Gaia DR2 5290850609994130560: in this case we defined a knot every 13-hours (grey lines in panel (a)).

We performed the procedure that we are going to describe considering each sector independent from the others; in a second step we performed again the same procedure for that stars observed in more than one sector and considering the entire light curve.

For the flattened light curves, we extracted the Transit- 

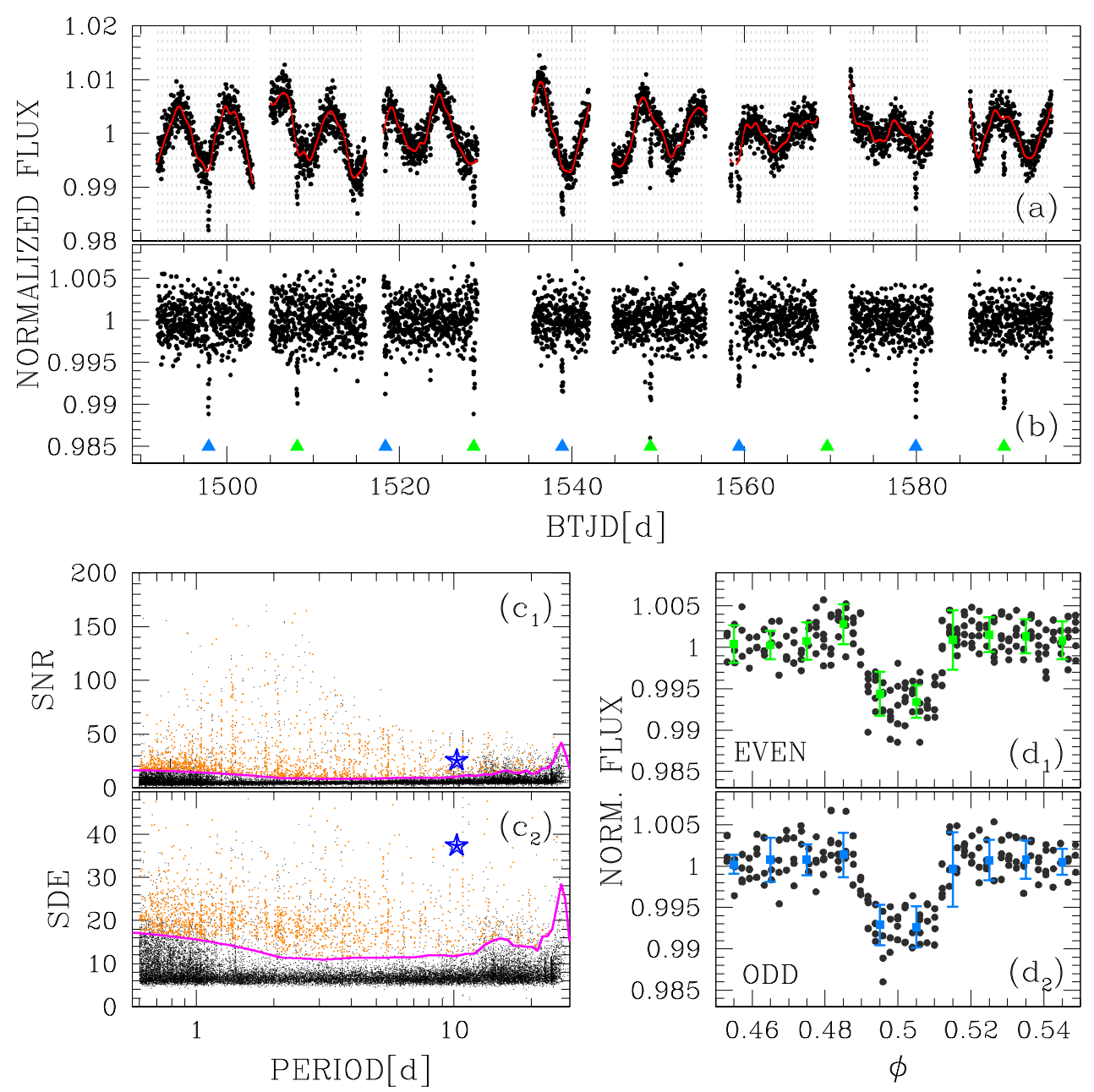

Figure 5. Overview of the successive steps of the procedure adopted to select candidate transiting objects: panel (a) shows the normalised light curve of Gaia DR2 5290850609994130560 from Sectors 7, 8, 9, and 10. The model (in red) is obtained interpolating a 5th order spline on a grid of knots spaced every 13-hours (grey lines). Panel (b) is the same light curve after removing the variability of the star: azure/green arrows are the odd/even transit events, found extracting the TLS periodogram. Panels (c) illustrate the procedure of selection of stars on the basis of the parameters extracted with the TLS routine: black points are all the stars observed in more than 1 sector, magenta lines are the limits above which stars pass the selection based on SDE/SNR parameters, orange points are the stars that pass all the selections (SDE/SNR/depth-of-transit/ $\left.\sigma_{\text {odd-even }}\right)$, and blue star is Gaia DR2 5290850609994130560. Panels $\left(\mathrm{d}_{1}\right)$ and $\left(\mathrm{d}_{2}\right)$ are the phased even and odd transits, respectively. Green and azure points are the means (and their standard deviations) of the phased points calculated in bins of width 0.01. Comparison of even/odd transit depths is useful to discriminate between candidate exoplanets and eclipsing binaries (see text for details).

fitting Least Squares (TLS) periodograms ${ }^{4}$ (Hippke \& Heller 2019), searching for transiting objects having period between $0.6 \mathrm{~d} \leq P \leq 0.5 \times T_{\mathrm{LC}}$, where $T_{\mathrm{LC}}$ is the maximum temporal baseline of the light curve (usually, for a single sector $T_{\mathrm{LC}} \sim 27$ days). In addition to the periodograms, the routine extracted many parameters useful to discriminate between light curves with/without transit signals, such as: the signal detection efficiency (SDE), the signal-to-noise ratio (SNR), the depth of the transit, and the significance between odd and even transit depths $\left(\sigma_{\text {odd-even }}\right)$. We used these parameters to perform a first selection of candidates: we divided the SDE and SNR distributions in intervals $\delta P=0.5 \mathrm{~d}$, and within each of them we calculated their $3.5 \sigma$-clipped

4 TLS v.1.0.24, https://github.com/hippke/tls mean (SD̄E and SNRR) and standard deviation $\left(\sigma_{\mathrm{S} D \bar{E}}\right.$ and $\left.\sigma_{\mathrm{SN} R}\right)$. We interpolated SD̄E $+3.5 \sigma_{\mathrm{S} \overline{\mathrm{DE}}}$ and $\mathrm{S} \overline{\mathrm{N} R}+3.5 \sigma_{\mathrm{SN} R}$ with splines, and we saved all the light curves which SDE and SNR values are above these splines, depth $<10 \%$, and $\sigma_{\text {odd-even }}<2.5$. This selection procedure is shown in panels $\left(c_{1}\right)$ and $\left(c_{2}\right)$ of Fig. 5: black points are all the stars for which we extracted the periodograms and that are observed in more than one sector, magenta line is the lower boundary for SNR and SDE selections, orange points are the stars that passed all the above described selections, and blue star is the candidate Gaia DR2 5290850609994130560. On average, the number of stars that passed these selection is $\sim 3 \%$ of the total number of analysed light curves. After this selection, in order to avoid false positive due to blends, we identified the groups of stars which present similar period 

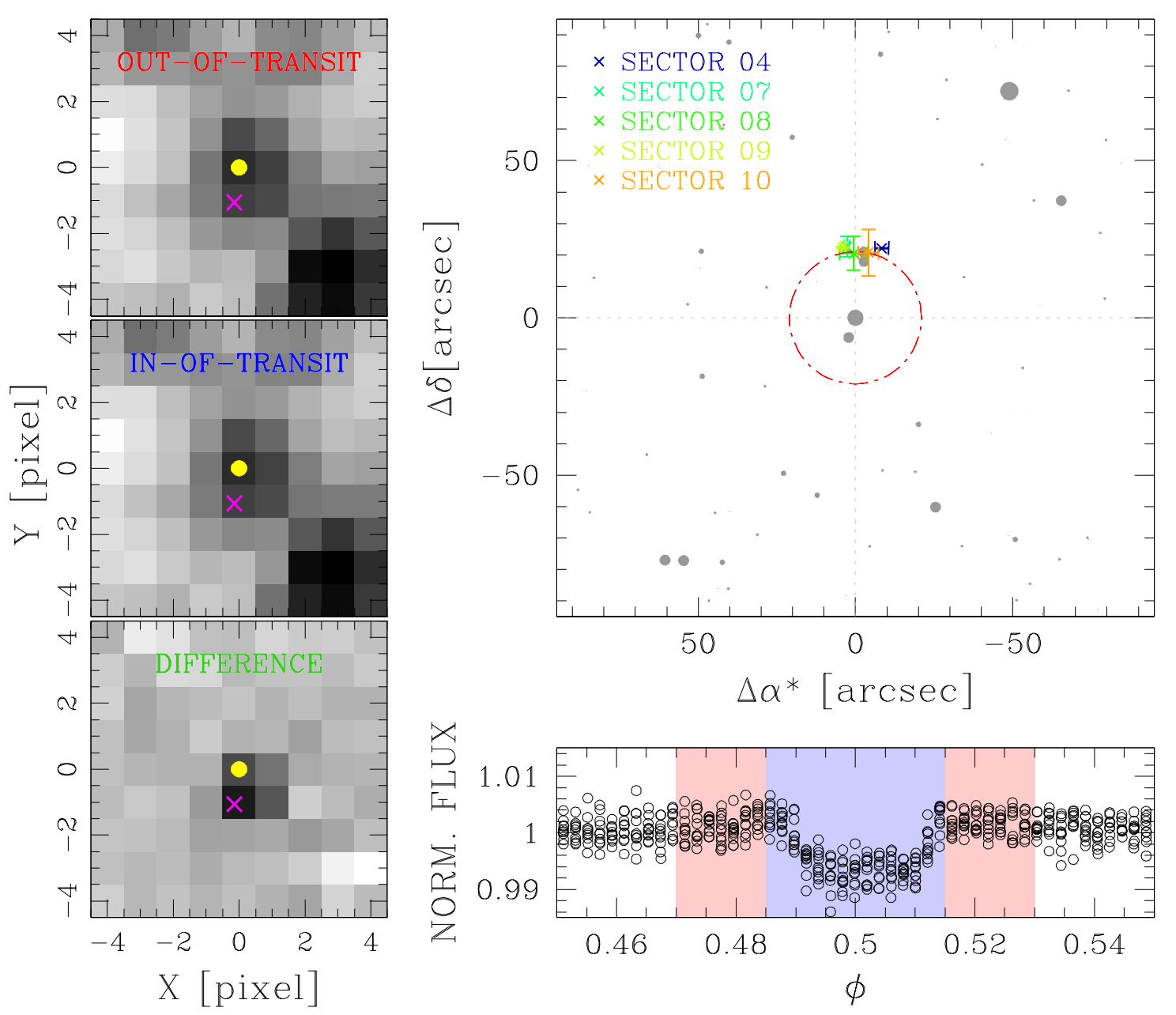

Figure 6. Vetting procedure of the candidate Gaia DR2 5290850609994130560 based on the position of the out-/in-of-transit difference image centroid. Left panels show the average of the images corresponding to out-of-transit (top panel) and in-of-transit (middle panel) points, and the difference between these two images (bottom panel); yellow circle is the position of the target star as reported in the Gaia DR2 catalogue, while magenta cross is the photocenter calculated on the difference image. Top right-hand panel shows the $95 \times 95$ arcsec ${ }^{2}$ finding chart centred on the target star and based on the Gaia DR2 catalogue. The crosses, colour-coded as in the legenda, are the mean offsets calculated for each sector in which the star is observed; red circle represents the photometric aperture adopted to analyse the light curve. Bottom right-hand panel is the phased light curve of the target star: light red and blue regions contain the out- and in-of-transit points, respectively.

within $0.05 \mathrm{~d}$ and they are separated by $<5$ pixels, and we exclude all these stars if one of them shows a signal that can not be associated to a transiting planet (e.g., a too deep transit or the presence of a secondary eclipse). Finally, we visually inspected the remaining light curves to check the odd/even transit depths (like in panels (d) of Fig. 5), the presence of artifacts that generate false transits, and the presence of secondary eclipses. On average, the final list of candidate transiting object contains $\sim 0.1 \%$ of the analysed objects; these candidates went through of a detailed vetting procedure to exclude possible remaining false positives.

\subsection{Vetting of the candidates}

We verified that the objects that survived to the previously described selections are genuine candidates, checking if the transit signals are due to a neighbour source. In order to verify this hypothesis, we performed three different tests.
For the first test, we verified if the transit depth of the candidate object changes considering light curves obtained with different photometric methods. For example, if the transit events are due to a neighbour eclipsing binary, we expect that large photometric apertures give deeper transit events. Moreover, as already demonstrated in Paper I, PSFfitting photometry is less affected by contamination than aperture photometry and allows us to discriminate between true and false positives. To perform this test, we estimated the mean value (and its standard deviation $\sigma$ ) of the transit depth for each photometric method; if the transit associated to a photometric method is $2 \sigma$ deeper than the mean transit depth associated to the analysed light curve, we considered the star as false positive.

The second test consisted in checking the binned phased light curve with a period equal to $0.5 \times, 1 \times$ and $2 \times$ the period found by TLS, and searching for secondary eclipses outside 
the primary transit. A star is considered false positive if the mean depths of even/odd transits differ $>2 \sigma$.

Finally, we computed the in/out-of-transit difference centroid to check if the transit events are associated to the target or to a close star. To calculate the centroid, we followed the procedure illustrated in Fig. 6 in the case of the star Gaia DR2 5290850609994130560: we identified the FFIs corresponding to the in-of-transit and out-of-transit points of the light curve, (light blue and light red shaded regions in the bottom-right panel of Fig. 6, respectively). For each transit, we calculated the mean out-of-transit image, the mean in-of-transit image, and their difference (top, middle, and bottom left-hand panels of Fig. 6, respectively). For each transit, we then calculated the photocenter on the difference image (magenta cross) and its offset relative to the Gaia DR2 position of the target star (yellow circle). Finally, for each sector, we calculated the mean value and its standard deviation of all the offsets. Top right-hand panel shows the finding chart $\left(95 \times 95 \operatorname{arcsec}^{2}\right)$ based on the Gaia DR2 catalogue and centred on Gaia DR2 5290850609994130560: the average offsets for all the sectors in which the star is observed are all located at a distance $\sim 20$ arcsec from the target star, in a region corresponding to two close stars of magnitude 14.5 (Gaia DR2 5290850609994130944 and Gaia DR2 5290850605693476608). Therefore, we discarded this candidate because the transit signals are not associated to the cluster member, but to a background star close to our target.

\subsection{TESS Objects of Interest}

We cross-matched our catalogue of analysed cluster members with the list of the TESS Objects of Interest ${ }^{5}$ (TOIs) released by the TESS team. We found 5 stars in common: three of them (TOI-496, TOI-681, and TOI-837) are also detected by our pipeline (PATHOS-6, PATHOS-25, and PATHOS$30)$. The other two TOIs were initially detected in the first selection of candidates we did, and then excluded by our vetting: (1) TOI-517 resulted to be an eccentric eclipsing binary with period $P \sim 9.885 \mathrm{~d}$ (as also reported in the public comments of the TOI); (2) from the analysis of the in-/outof-transit centroid of TOI-861 we found that the signal is associated to a close star (TIC 0372913432) at $\sim 40 \operatorname{arcsec}$ from the target, and that this star is catalogued as binary by Bailey et al. (2018).

\section{MODELLING OF THE TRANSITS}

In our final catalogue of genuine transiting objects there are 33 sources associated to 28 stellar clusters. For the extraction of the physical parameters (that can be obtained from a light curve analyses) of the transiting objects we need to know the stellar parameters. As stars in open clusters are located at the same distance and have the same chemical composition and age, we can use theoretical models to estimate their main parameters including temperature, mass and radius. In the next section, we describe how we extracted these, that will be then used as priors for the modelling of the transits (Sect. 4.2).

\subsection{Stellar parameters}

We extracted the information on the cluster members that host candidate transiting exoplanets by fitting isochrones to the colour-magnitude diagrams (CMDs) of each stellar cluster and deriving the stellar parameters (mass, effective temperature, luminosity, and radius) interpolating colour and magnitude of each star on the isochrone. In this work we used the last release of BaSTI ("a Bag of Stellar Tracks and Isochrones") models (Hidalgo et al. 2018) to fit isochrones on the CMDs. In order to perform this fitting, it is necessary to know the age, metallicity, distance, and reddening of the stellar clusters.

Unfortunately, metallicity measurements are not available for the large part of the stellar clusters in our sample. For this reason, we considered a constant metallicity $[\mathrm{Fe} / \mathrm{H}]=0.0$ for all the clusters and, at the end, we added to the final error on stellar parameters, the contribution of considering a wrong $[\mathrm{Fe} / \mathrm{H}]$ in the isochrone fitting procedure. The corresponding uncertainty has been estimated using 3 different isochrones with age of $300 \mathrm{Myr}$ (the average age of the studied clusters), but with metallicity $[\mathrm{Fe} / \mathrm{H}]=-0.3$, $[\mathrm{Fe} / \mathrm{H}]=0.0$, and $[\mathrm{Fe} / \mathrm{H}]=+0.3$, that is the range of metallicities spanned by the Galactic stellar clusters for which there are $[\mathrm{Fe} / \mathrm{H}]$ measurements. We found that, for main sequence stars with mass $0.5 M_{\odot}<M_{\star}<1.5 M_{\odot}$, the mean differences on the stellar radius $R_{\star}$, mass $M_{\star}$, and effective temperature $T_{\text {eff }}$ between isochrones with $[\mathrm{Fe} / \mathrm{H}]=0.0$ and $[\mathrm{Fe} / \mathrm{H}]= \pm 0.3$ are $\sim 0.03 \mathrm{R}_{\odot}, \sim 0.05 \mathrm{M}_{\odot}$, and $\sim 200 \mathrm{~K}$, respectively.

We extracted the information on the distance of the clusters from Cantat-Gaudin et al. (2018), while ages and reddenings are extracted from two different catalogues: for 13 stellar clusters we used the cluster parameters and the associated errors found by Bossini et al. (2019) analysing the Gaia DR2 CMDs; for the other clusters we used the information given by Kharchenko et al. (2016). In the latter catalogue the errors on age and reddening are not given. In this case, we adopted an error equal to $10 \%$ of the measurement. Muzzio 1 is the only cluster that is not present in both catalogues; for this very young cluster we used the information given by Bica \& Bonatto (2011).

Table 1 lists the cluster parameters. Thanks to isochrone fits, we were able to extract the stellar parameters of the cluster members that host candidate transiting exoplanets. These parameters were then used as priors in the phase of modelling described in the next section. Isochrone fits are shown in Figs. A1, A2, and A3.

\subsection{Transit modelling}

For the transit modelling and analysis, we made use of PyORBIT $^{6}$ (Malavolta et al. 2016, 2018; see also, e.g., Benatti et al. 2019). The routine is a wrapper for the transit modelling code batman (Kreidberg 2015) and the affine invariant Markov chain Monte Carlo (MCMC) sampler emcee 
Table 1. Cluster parameters

\begin{tabular}{lccccc}
\hline Cluster Name & $\begin{array}{c}\text { Age } \\
(\mathrm{Myr})\end{array}$ & $\begin{array}{c}\text { Distance } \\
(\mathrm{pc})\end{array}$ & $E(B-V)$ & $\begin{array}{c}\pi^{(\mathrm{a})} \\
(\mathrm{mas})\end{array}$ & Reference \\
\hline Alessi 8 & $137 \pm 35$ & $663.7 \pm 9.5$ & $0.10 \pm 0.01$ & $1.479 \pm 0.046$ & $(2)$ \\
ASCC 85 & $32 \pm 3$ & $870.0 \pm 5.1$ & $0.52 \pm 0.05$ & $1.119 \pm 0.085$ & $(2)$ \\
ASCC 88 & $20 \pm 2$ & $883.3 \pm 5.5$ & $0.73 \pm 0.07$ & $1.097 \pm 0.060$ & $(2)$ \\
Collinder 292 & $498 \pm 52$ & $1461.5 \pm 16.9$ & $0.29 \pm 0.01$ & $0.528 \pm 0.045$ & $(1)$ \\
Haffner 14 & $195 \pm 10$ & $4359.1 \pm 38.4$ & $0.61 \pm 0.01$ & $0.226 \pm 0.041$ & $(1)$ \\
Harvard 5 & $65 \pm 34$ & $1203.9 \pm 15.6$ & $0.22 \pm 0.01$ & $0.764 \pm 0.044$ & $(1)$ \\
IC 2602 & $35 \pm 2$ & $152.3 \pm 1.0$ & $0.03 \pm 0.01$ & $6.561 \pm 0.157$ & $(1)$ \\
Melotte 101 & $125 \pm 13$ & $2134.7 \pm 9.2$ & $0.45 \pm 0.05$ & $0.439 \pm 0.048$ & $(2)$ \\
Muzzio 1 & $5 \pm 4$ & $1824.8 \pm 24.3$ & $1.02 \pm 0.56$ & $0.519 \pm 0.023$ & $(3)$ \\
NGC 2112 & $2065 \pm 207$ & $1103.6 \pm 3.6$ & $0.63 \pm 0.06$ & $0.877 \pm 0.066$ & $(2)$ \\
NGC 2318 & $560 \pm 56$ & $1342.9 \pm 8.5$ & $0.44 \pm 0.04$ & $0.716 \pm 0.042$ & $(2)$ \\
NGC 2323 & $95 \pm 10$ & $921.3 \pm 6.8$ & $0.15 \pm 0.01$ & $0.997 \pm 0.057$ & $(2)$ \\
NGC 2437 & $220 \pm 22$ & $1582.3 \pm 3.9$ & $0.15 \pm 0.01$ & $0.603 \pm 0.060$ & $(2)$ \\
NGC 2516 & $251 \pm 5$ & $415.1 \pm 1.0$ & $0.09 \pm 0.01$ & $2.417 \pm 0.045$ & $(1)$ \\
NGC 2527 & $830 \pm 48$ & $572.0 \pm 5.6$ & $0.06 \pm 0.01$ & $1.536 \pm 0.070$ & $(1)$ \\
NGC 2548 & $525 \pm 53$ & $758.8 \pm 2.0$ & $0.04 \pm 0.01$ & $1.289 \pm 0.065$ & $(2)$ \\
NGC 2669 & $82 \pm 3$ & $1124.1 \pm 10.4$ & $0.20 \pm 0.01$ & $0.845 \pm 0.046$ & $(1)$ \\
NGC 2671 & $338 \pm 34$ & $1396.5 \pm 7.2$ & $0.98 \pm 0.10$ & $0.687 \pm 0.031$ & $(2)$ \\
NGC 3114 & $200 \pm 20$ & $1017.3 \pm 1.1$ & $0.08 \pm 0.01$ & $0.954 \pm 0.048$ & $(2)$ \\
NGC 3532 & $399 \pm 10$ & $485.3 \pm 1.0$ & $0.02 \pm 0.01$ & $2.066 \pm 0.062$ & $(1)$ \\
NGC 5316 & $152 \pm 5$ & $1437.5 \pm 12.6$ & $0.25 \pm 0.01$ & $0.665 \pm 0.055$ & $(1)$ \\
NGC 5925 & $180 \pm 18$ & $1413.2 \pm 6.2$ & $0.75 \pm 0.07$ & $0.679 \pm 0.050$ & $(1)$ \\
Ruprecht 48 & $398 \pm 40$ & $3784.4 \pm 79.5$ & $0.28 \pm 0.03$ & $0.235 \pm 0.038$ & $(2)$ \\
Ruprecht 82 & $353 \pm 47$ & $2206.0 \pm 24.5$ & $0.33 \pm 0.02$ & $0.430 \pm 0.038$ & $(1)$ \\
Ruprecht 94 & $20 \pm 2$ & $2511.5 \pm 29.5$ & $0.05 \pm 0.05$ & $0.369 \pm 0.031$ & $(2)$ \\
Ruprecht 151 & $411 \pm 25$ & $1179.8 \pm 25.2$ & $0.19 \pm 0.01$ & $0.867 \pm 0.056$ & $(1)$ \\
SAI 91 & $676 \pm 68$ & $3571.6 \pm 90.7$ & $0.44 \pm 0.08$ & $0.251 \pm 0.040$ & $(2)$ \\
Trumpler 22 & $25 \pm 15$ & $2364.8 \pm 24.1$ & $0.59 \pm 0.01$ & $0.386 \pm 0.028$ & $(1)$ \\
\hline (1) Bossini et al. (2019); (2) Kharchenko et al. $(2016) ;(3)$ Bica \& Bonatto (2011) & \\
(a) Mean cluster parallax from Cantat-Gaudin et al. (2018) & & \\
& & & & &
\end{tabular}

(Foreman-Mackey et al. 2013) combined with global optimization algorithm $\mathrm{PyDE}^{7}$. We included in the transit model: the central time of the first transit $\left(T_{0}\right)$, the period $(P)$, the impact parameter $(b)$, the planetary to stellar radius ratio $\left(R_{\mathrm{p}} / R_{\star}\right)$, and the stellar density $\left(\rho_{\star}\right)$. For the modelling, we adopted a Keplerian orbit with null eccentricity. In Table 2 we listed the priors used for the fit. We used the $T_{\text {eff }}$ and $\log (g)$ values obtained with isochrones fitting to extract, through a bilinear interpolation, the priors on the limb darkening (LD) coefficients from the grid of values published by Claret (2018); for the modelling we adopted the LD parametrisation used by Kipping (2013). In the modelling process, we took into account of the 30-minute cadence of the TESS time-series (Kipping 2010). The routine explored all the parameters in linear space. For our fit, we made use of a number of walkers $N_{\text {walkers }}=110$, equal to 10 times the number of free parameters. We ran, for each model, the sampler for 50000 steps, removing the first 15000 steps as burn-in and using a thinning factor of 100 .

In Appendix A are all results: in Table A1 are listed the outputs of our fit and the clusters associated to each transiting object of interest; Figs. A1, A2, and A3 show, for each transiting object, the position of the star on the Gaia DR2 CMD of the cluster to which it is associated (lefthand panel), the folded light curve with the overimposed model of the transit (top right-hand panel), the difference between the observed points and the model (middle righthand panel), the finding chart and the vector-point diagram of the target, and its surrounding sources from the Gaia DR2 catalogue (bottom right-hand panels).

\section{EXOPLANETS IN OPEN CLUSTERS: RESULTS}

From our dataset, we extracted 33 objects of interest whose light curves show transit signals. In this section we isolate

\footnotetext{
7 https://github.com/hpparvi/PyDE
}

the more significant candidate exoplanets and compare the candidate exoplanets and cluster parameters.

From our final list, we excluded all stars that host candidate planets with a radius $R_{\mathrm{P}} \geq 3.0 R_{\mathrm{J}}$ ( 15 objects), because of their doubtful planet nature. We also excluded all those stars that have a parallax that differs $>3 \sigma$ from the mean parallax of the cluster (see Table 1), stars that are not located on the main sequence in the CMD of the cluster (i.e., $>2 \sigma$ from the mean colour of the main sequence), and stars whose proper motion differs by $>4 \sigma$ from the mean proper motion of the cluster. In this way, we were left with 11 candidates (PATHOS-3, 6, 8, 9, 15, 20, 21, 23, 25, 30, 31) in 8 open clusters, 7 of them (NGC 2112, NGC 2437, NGC 2516, NGC 2527, NGC 2548, NGC 3532, and IC 2602) having a solar metallicity (Netopil et al. 2016), while for NGC 2323 there are no metallicity measurements; 9 of these candidate exoplanets orbit stars with radii $R_{\star} \lesssim 1.5 R_{\odot}$, while PATHOS-20 and PATHOS-23 orbit stars with $R_{\star} \sim 2.2 R_{\odot}$ and $R_{\star} \sim 2.5 R_{\odot}$, respectively.

Figure 7 shows the correlations between cluster age and stellar density and some candidate exoplanet parameters, like period, semi-major axis, and radius. In this analysis we also included the candidate and confirmed transiting exoplanets in open clusters and young association found by Kepler/K2 and TESS, present in literature. In particular, we considered the first two discovered exoplanets (Kepler66b and Kepler-67b, Meibom et al. 2013) in NGC 6811 ( $863 \mathrm{Myr}$ ), the 4 exoplanets K2-25b, K2-136Ab,c,d (Ciardi et al. 2018; Mann et al. 2018) in the Hyades ( $730 \mathrm{Myr})$, the 6 exoplanets in M 44 ( 670 Myr), e.g., K2-95b, K2-100b, K2-101b, K2-102b, K2-103b, K2-104b (Barros et al. 2016; Libralato et al. 2016b; Pope et al. 2016; Obermeier et al. 2016; Pepper et al. 2017; Mann et al. 2017), and the Ruprecht 147 ( 3 Gyr) member K2-213b (Curtis et al. 2018). In our analysis, we also considered the exoplanets K2-33b in the young association Upper Scorpius ( $10 \mathrm{Myr}$, David et al. 2016b; Mann et al. 2016a), EPIC 247267267 b in the CasTau group ( $120 \mathrm{Myr}$, David et al. 2018) and the recently discovered exoplanets DS Tuc Ab (Newton et al. 2019; Benatti et al. 2019), discovered by TESS and member of the Tucana-Horologium young association $(\sim 40 \mathrm{Myr})$.

Blue points and histograms in Fig. 7 correspond to the objects identified in this work; red points and histograms are the planets from Kepler observations; green points and histograms represent stars in young associations. Considering all the points, there is no evident correlations among cluster/stellar and (candidate) exoplanet parameters. Figure 7 illustrates the difference between the type of exoplanets in stellar clusters detected by Kepler and TESS: the large part of the candidates found in this work are Jupiter-size objects orbiting stars with $0.7 R_{\odot} \lesssim R_{\star} \lesssim 2.5 R_{\odot}$, while the transiting objects found with Kepler and K2 data of open clusters are Neptune- and Earth-size exoplanets hosted by $R_{\star} \lesssim 1 R_{\odot}$ stars. This is mainly due to the combination of two observational biases: (i) Kepler data limiting magnitude is about 2-3 magnitudes fainter than TESS one, and therefore, at a given magnitude, Kepler photometric precision is higher than TESS one, allowing us to find smaller exoplanets around fainter main sequence stars; (ii) 3 of the 4 open clusters (Hyades, M 44, and Ruprecht 147) observed by Kepler and whose members host known exoplanets are "close" clusters $(d \lesssim 300$ pc, while NGC 6811 is at $\sim 1250$ pc), 
Table 2. Star parameters and priors for the modelling

\begin{tabular}{|c|c|c|c|c|c|c|c|c|c|c|c|c|c|}
\hline TIC & PATHOS & Cluster & $\begin{array}{c}\alpha \\
(\mathrm{deg} .)\end{array}$ & $\begin{array}{c}\delta \\
(\mathrm{deg} .)\end{array}$ & $\begin{array}{c}\pi \\
\text { (mas) }\end{array}$ & $\begin{array}{c}T \\
\text { (mag.) }\end{array}$ & 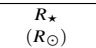 & $\begin{array}{c}M_{\star} \\
\left(M_{\odot}\right)\end{array}$ & $\begin{array}{c}\rho_{\star} \\
\left(\rho_{\odot}\right)\end{array}$ & $\begin{array}{l}\text { Period } \\
\text { (d) }\end{array}$ & $\begin{array}{c}T_{0} \\
\text { (BTJD) }\end{array}$ & $\mathrm{LD}_{c 1}$ & $\mathrm{LD}_{c 2}$ \\
\hline 0030654608 & 2 & Muzzio 1 & 134.3845 & -47.8477 & 0.5191 & 10.4 & $10.0 \pm 2.00$ & $17.0 \pm 2.00$ & $\mathcal{N}(0.01,0.10)$ & $\mathcal{U}(2.0,3.0)$ & $\mathcal{U}(1519.0,1520.0)$ & $\mathcal{N}(0.10,0.50)$ & $\mathcal{N}(0.10,0.50)$ \\
\hline 0039291805 & 3 & NGC 2112 & 88.7164 & -0.0484 & 0.8964 & 15.2 & $1.12 \pm 0.03$ & $1.13 \pm 0.05$ & $\mathcal{N}(0.80,0.10)$ & $\mathcal{U}(7.0,7.5)$ & $\mathcal{U}(1468.9,1469.2)$ & $\mathcal{N}(0.31,0.05)$ & $\mathcal{N}(0.29,0.05)$ \\
\hline 0042524156 & 4 & $\operatorname{ASCC} 88$ & 256.8882 & -35.5036 & 1.1502 & 10.5 & $2.94 \pm 0.03$ & $5.18 \pm 0.05$ & $\mathcal{N}(0.20,0.10)$ & $\mathcal{U}(9.8,10.2)$ & $\mathcal{U}(1631.0,1632.0)$ & $\mathcal{N}(0.15,0.13)$ & $\mathcal{N}(0.15,0.13)$ \\
\hline 0080317933 & 5 & Collinder 292 & 237.9156 & -57.2797 & 0.6320 & 12.9 & $1.83 \pm 0.03$ & $1.91 \pm 0.05$ & $\mathcal{N}(0.32,0.10)$ & $\mathcal{U}(3.0,3.5)$ & $\mathcal{U}(1625.0,1626.0)$ & $\mathcal{N}(0.19,0.05)$ & $\mathcal{N}(0.23,0.05)$ \\
\hline 0088977253 & 6 & NGC 2548 & 123.0977 & -5.7687 & 1.2358 & 12.3 & $1.42 \pm 0.03$ & $1.42 \pm 0.05$ & $\mathcal{N}(0.49,0.10)$ & $\mathcal{U}(2.5,3.0)$ & $\mathcal{U}(1492.0,1493.0)$ & $\mathcal{N}(0.27,0.05)$ & $\mathcal{N}(0.28,0.05)$ \\
\hline 0092835691 & 7 & SAI 91 & 129.1193 & -50.1497 & 0.2347 & 14.6 & $2.24 \pm 0.05$ & $2.02 \pm 0.07$ & $\mathcal{N}(0.18,0.10)$ & $\mathcal{U}(22.0,23.0)$ & $\mathcal{U}(1527.0,1528.0)$ & $\mathcal{N}(0.19,0.05)$ & $\mathcal{N}(0.23,0.05)$ \\
\hline 0094589619 & 8 & NGC 2437 & 115.7338 & -14.5810 & 0.6238 & 13.8 & $1.46 \pm 0.03$ & $1.55 \pm 0.05$ & $\mathcal{N}(0.49,0.10)$ & $\mathcal{U}(11.8,12.5)$ & $\mathcal{U}(1496.0,1497.0)$ & $\mathcal{N}(0.24,0.05)$ & $\mathcal{N}(0.26,0.05)$ \\
\hline 0125414447 & 9 & NGC 2323 & 105.7303 & -8.4369 & 0.9320 & 14.2 & $1.00 \pm 0.03$ & $1.10 \pm 0.05$ & $\mathcal{N}(1.10,0.10)$ & $\mathcal{U}(3.5,4.0)$ & $\mathcal{U}(1493.0,1494.0)$ & $\mathcal{N}(0.32,0.05)$ & $\mathcal{N}(0.30,0.05)$ \\
\hline 0126600730 & 10 & Haffner 14 & 116.0772 & -28.3253 & 0.2145 & 15.4 & $2.11 \pm 0.03$ & $2.56 \pm 0.05$ & $\mathcal{N}(0.27,0.10)$ & $\mathcal{U}(6.0,6.5)$ & $\mathcal{U}(1497.0,1498.0)$ & $\mathcal{N}(0.15,0.05)$ & $\mathcal{N}(0.20,0.05)$ \\
\hline 0144995073 & 11 & NGC 2669 & 131.4641 & -52.9284 & 0.8289 & 13.7 & $1.36 \pm 0.03$ & $1.41 \pm 0.05$ & $\mathcal{N}(0.55,0.10)$ & $\mathcal{U}(19.5,20.5)$ & $\mathcal{U}(1518.5,1519.5)$ & $\mathcal{N}(0.27,0.05)$ & $\mathcal{N}(0.28,0.05)$ \\
\hline 0147069011 & 12 & Alessi 8 & 232.8684 & -51.1783 & 1.5017 & 12.5 & $1.25 \pm 0.03$ & $1.30 \pm 0.05$ & $\mathcal{N}(0.66,0.10)$ & $\mathcal{U}(7.0,7.5)$ & $\mathcal{U}(1628.0,1629.0)$ & $\mathcal{N}(0.28,0.05)$ & $\mathcal{N}(0.29,0.05)$ \\
\hline 0147426828 & 13 & NGC 2318 & 104.8768 & -13.2535 & 0.6934 & 14.1 & $1.49 \pm 0.03$ & $1.49 \pm 0.05$ & $\mathcal{N}(0.45,0.10)$ & $\mathcal{U}(1.0,1.7)$ & $\mathcal{U}(1492.0,1493.0)$ & $\mathcal{N}(0.25,0.05)$ & $\mathcal{N}(0.27,0.05)$ \\
\hline 0153734545 & 14 & NGC 2527 & 121.2402 & -28.1462 & 1.5019 & 9.9 & $2.72 \pm 0.03$ & $2.05 \pm 0.05$ & $\mathcal{N}(0.11,0.10)$ & $\mathcal{U}(3.0,3.5)$ & $\mathcal{U}(1493.0,1494.0)$ & $\mathcal{N}(0.22,0.05)$ & $\mathcal{N}(0.25,0.05)$ \\
\hline 0153735144 & 15 & NGC 2527 & 121.1537 & -28.2948 & 1.5432 & 11.6 & $1.51 \pm 0.03$ & $1.46 \pm 0.05$ & $\mathcal{N}(0.43,0.10)$ & $\mathcal{U}(3.2,4.0)$ & $\mathcal{U}(1493.0,1494.0)$ & $\mathcal{N}(0.26,0.05)$ & $\mathcal{N}(0.27,0.05)$ \\
\hline 0159059181 & 16 & NGC 2112 & 88.3711 & 0.4239 & 0.8356 & 14.2 & $1.52 \pm 0.03$ & $1.33 \pm 0.05$ & $\mathcal{N}(0.38,0.10)$ & $\mathcal{U}(5.0,5.5)$ & $\mathcal{U}(1470.9,1471.4)$ & $\mathcal{N}(0.28,0.05)$ & $\mathcal{N}(0.28,0.05)$ \\
\hline 0181602717 & 17 & NGC 2671 & 131.7366 & -41.9773 & 0.5930 & 15.2 & $1.47 \pm 0.03$ & $1.53 \pm 0.05$ & $\mathcal{N}(0.48,0.10)$ & $\mathcal{U}(2.0,2.7)$ & $\mathcal{U}(1517.0,1518.0)$ & $\mathcal{N}(0.24,0.05)$ & $\mathcal{N}(0.25,0.05)$ \\
\hline 0236084210 & 18 & $\mathrm{ASCC} 85$ & 251.5388 & -45.3214 & 1.1433 & 11.5 & $1.84 \pm 0.03$ & $2.66 \pm 0.05$ & $\mathcal{N}(0.42,0.10)$ & $\mathcal{U}(5.0,5.5)$ & $\mathcal{U}(1631.5,1632.5)$ & $\mathcal{N}(0.15,0.05)$ & $\mathcal{N}(0.18,0.05)$ \\
\hline 0300362600 & 19 & NGC 5316 & 208.3975 & -62.0764 & 0.6984 & 12.2 & $2.14 \pm 0.03$ & $2.70 \pm 0.05$ & $\mathcal{N}(0.28,0.10)$ & $\mathcal{U}(11.0,12.0)$ & $\mathcal{U}(1601.9,1602.5)$ & $\mathcal{N}(0.15,0.05)$ & $\mathcal{N}(0.19,0.05)$ \\
\hline 0306385801 & 20 & NGC 3532 & 166.6553 & -58.8608 & 2.0510 & 9.5 & $2.22 \pm 0.03$ & $2.29 \pm 0.05$ & $\mathcal{N}(0.21,0.10)$ & $\mathcal{U}(13.8,14.5)$ & $\mathcal{U}(1574.8,1575.3)$ & $\mathcal{N}(0.16,0.05)$ & $\mathcal{N}(0.21,0.05)$ \\
\hline 0308538095 & 21 & NGC 2516 & 120.8514 & -60.6655 & 2.4333 & 11.6 & $1.29 \pm 0.03$ & $1.32 \pm 0.05$ & $\mathcal{N}(0.62,0.10)$ & $\mathcal{U}(11.0,12.5)$ & $\mathcal{U}(1420.0,1421.0)$ & $\mathcal{N}(0.29,0.05)$ & $\mathcal{N}(0.29,0.05)$ \\
\hline 0317536999 & 22 & Ruprecht 94 & 172.5414 & -63.1323 & 0.3921 & 14.1 & $1.86 \pm 0.03$ & $2.76 \pm 0.05$ & $\mathcal{N}(0.43,0.10)$ & $\mathcal{U}(5.5,6.0)$ & $\mathcal{U}(1575.5,1576.5)$ & $\mathcal{N}(0.15,0.05)$ & $\mathcal{N}(0.17,0.05)$ \\
\hline 0372913337 & 23 & NGC 2516 & 119.4918 & -60.8459 & 2.3454 & 8.9 & $2.49 \pm 0.03$ & $2.74 \pm 0.05$ & $\mathcal{N}(0.18,0.10)$ & $\mathcal{U}(1.5,2.0)$ & $\mathcal{U}(1325.0,1326.0)$ & $\mathcal{N}(0.15,0.05)$ & $\mathcal{N}(0.19,0.05)$ \\
\hline 0389927567 & 24 & Melotte 101 & 160.2669 & -65.5215 & 0.4321 & 13.0 & $2.26 \pm 0.03$ & $2.95 \pm 0.05$ & $\mathcal{N}(0.25,0.10)$ & $\mathcal{U}(6.5,7.0)$ & $\mathcal{U}(1575.0,1576.0)$ & $\mathcal{N}(0.15,0.05)$ & $\mathcal{N}(0.17,0.05)$ \\
\hline 0410450228 & 25 & NGC 2516 & 117.8949 & -60.4124 & 2.3447 & 10.6 & $1.51 \pm 0.03$ & $1.67 \pm 0.05$ & $\mathcal{N}(0.48,0.10)$ & $\mathcal{U}(15.5,16.0)$ & $\mathcal{U}(1420.0,1421.0)$ & $\mathcal{N}(0.22,0.05)$ & $\mathcal{N}(0.25,0.05)$ \\
\hline 0413809436 & 26 & NGC 5925 & 232.0979 & -54.5586 & 0.6814 & 14.1 & $1.59 \pm 0.03$ & $1.88 \pm 0.05$ & $\mathcal{N}(0.47,0.10)$ & $\mathcal{U}(2.5,3.0)$ & $\mathcal{U}(1627.0,1628.0)$ & $\mathcal{N}(0.18,0.05)$ & $\mathcal{N}(0.23,0.05)$ \\
\hline 0419091401 & 27 & Ruprecht 48 & 120.6352 & -31.9951 & 0.2379 & 13.7 & $3.40 \pm 0.20$ & $2.71 \pm 0.10$ & $\mathcal{N}(0.07,0.10)$ & $\mathcal{U}(12.5,13.0)$ & $\mathcal{U}(1499.0,1500.0)$ & $\mathcal{N}(0.15,0.10)$ & $\mathcal{N}(0.21,0.10)$ \\
\hline 0432564189 & 28 & Ruprecht 82 & 146.5229 & -53.9787 & 0.4632 & 13.6 & $2.12 \pm 0.05$ & $2.28 \pm 0.05$ & $\mathcal{N}(0.23,0.10)$ & $\mathcal{U}(3.5,4.0)$ & $\mathcal{U}(1546.0,1547.0)$ & $\mathcal{N}(0.16,0.05)$ & $\mathcal{N}(0.21,0.05)$ \\
\hline 0450610413 & 29 & Harvard 5 & 186.7427 & -60.6906 & 0.6932 & 12.2 & $1.72 \pm 0.05$ & $2.30 \pm 0.05$ & $\mathcal{N}(0.45,0.10)$ & $\mathcal{U}(10.0,11.0)$ & $\mathcal{U}(1599.8,1600.8)$ & $\mathcal{N}(0.15,0.05)$ & $\mathcal{N}(0.20,0.05)$ \\
\hline 0460205581 & 30 & IC 2602 & 157.0373 & -64.5052 & 6.9893 & 9.9 & $1.07 \pm 0.03$ & $1.15 \pm 0.05$ & $\mathcal{N}(0.93,0.10)$ & $\mathcal{U}(8.0,8.5)$ & $\mathcal{U}(1574.0,1575.0)$ & $\mathcal{N}(0.31,0.05)$ & $\mathcal{N}(0.30,0.05)$ \\
\hline 0460950389 & 31 & IC 2602 & 159.1580 & -64.7982 & 6.6229 & 11.6 & $0.84 \pm 0.03$ & $0.81 \pm 0.05$ & $\mathcal{N}(1.37,0.10)$ & $\mathcal{U}(2.5,3.0)$ & $\mathcal{U}(1572.0,1573.0)$ & $\mathcal{N}(0.43,0.05)$ & $\mathcal{N}(0.38,0.05)$ \\
\hline 04620 & 32 & NGC 3114 & 149.9120 & -59.9032 & 1.0387 & 11.3 & $2.01 \pm 0.03$ & $2.43 \pm 0.05$ & $\mathcal{N}(0.2$ & $\mathcal{U}(2.0,2.5)$ & $\mathcal{U}(154$ & $\mathcal{N}(0.15,0.05)$ & $\mathcal{N}(0.20,0.05)$ \\
\hline 0748919024 & 33 & Ruprecht 151 & 115.1315 & -16 & 0.73 & 13.4 & $1.46 \pm 0.03$ & $1.49 \pm 0.05$ & $\mathcal{N}(0.48,0.10)$ & $\mathcal{U}(2.0,3.0)$ & $\mathcal{U}(1493.8,1494.5)$ & $\mathcal{N}(0.25,0.05)$ & $\mathcal{N}(0.27,0.05)$ \\
\hline 1036769612 & 34 & Trumpler 22 & 217.5064 & -61.3249 & 0.4244 & 15.8 & $1.43 \pm 0.05$ & $1.58 \pm 0.10$ & $\mathcal{N}(0.54,0.20)$ & $\mathcal{U}(7.1,7.7)$ & $\mathcal{U}(1599.0,1600.0)$ & $\mathcal{N}(0.24,0.05)$ & $\mathcal{N}(0.27,0.05)$ \\
\hline
\end{tabular}

while distances of the open clusters studied in this work are between $100 \mathrm{pc}$ and $15 \mathrm{kpc}$, with $50 \%$ of the open cluster having $d \gtrsim 3 \mathrm{kpc}$.

Figure 8 shows how the distance of the open clusters affects our results. We analysed what kind of planets we are able to detect in the light curves of stars having a radius $R_{\star}=0.5 R_{\odot}$ (top panel), $R_{\star}=1.0 R_{\odot}$ (middle panel), and $R_{\star}=1.5 R_{\odot}$ (bottom panel), and located at distances between 10 and 15000 pc. Using isochrones, we calculated the average absolute TESS magnitude of these stars and then we calculated their apparent magnitudes for different distances. The upper axis of each panel illustrates the apparent magnitude $T$ of a star at the corresponding distance of the lower axis. The grey zone represents the magnitude range out of reach of TESS. Red histogram is the distribution of the distances of the stars in the clusters studied in this work: the peak of the distribution is at $d \sim 3000 \mathrm{pc}$. Magenta line represents the mean distribution of the RMS calculated using all the analysed flattened light curves; the RMS gives us an idea about the detectability of an exoplanet of radius $R_{\mathrm{p}}$ hosted by a star of radius $R_{\star}$ located at distance $d$.

We analysed four different types of exoplanets orbiting stars into the three $R_{\star}$ groups: Earth-size planet $\left(R_{\mathrm{p}}=1 R_{\mathrm{E}}\right.$, green dot in Fig. 8), super-Earth-size planet $\left(R_{\mathrm{p}}=2 R_{\mathrm{E}}\right.$, orange dot in Fig. 8), Neptune-size planet $\left(R_{\mathrm{p}}=1 R_{\mathrm{N}}\right.$, light blue dot in Fig. 8), and Jupiter-size planet $\left(R_{\mathrm{p}}=1 R_{\mathrm{J}}\right.$, yellow dot in Fig. 8). We calculated the expected photometric decrement in the light curve $\delta_{\text {phot }}=\left(R_{\mathrm{P}} / R_{\star}\right)^{2}$ due to the exoplanet transit and, by using the photometric precision distribution, we calculated the maximum distance at which the transit of a planet of radius $R_{\mathrm{P}}$ is still detectable (at $1 \sigma$ ) in the light curve of a star with radius $R_{\star}$. Finally, we used this distance, combined with the CMD $M_{T}$ versus $\left(G_{\mathrm{BP}}-G_{\mathrm{RP}}\right)_{0}$, to extract the number $\left(N_{\star}\right)$ of cluster members having radius $R_{\star}$ for which this kind of transits can be detected in their light curves. This CMD is shown in Fig. 9. For the reddening correction, we used the tridimensional extinction map of Lallement et al. (2018) in conjunction with Bailer-Jones et al. (2018) distances to estimate the amount of interstellar reddening to the target stars. We evaluated the reddening $E(B-V)$ on a sample of $N$, equally spaced, nodal points along each target direction by considering the weighted average of the 27 surrounding voxels reddening $(3 \times 3 \times 3$ voxels cube around the nodal point). The reddening was weighted by the distance of the nodal point from each one of the neighbouring voxels' centers while the distance between two adjacent nodal points was $2 \mathrm{pc}$. We therefore summed up the reddening along the boresight to derive the integrated reddening to the target stars. For stars falling outside the map we added a correction to account for the extinction from the edge of the map to the target position. The correction was calculated using the dust model described in Binney et al. (2014). The entire procedure will be further described in Montalto et al. (in preparation). For each cluster, we calculated the $3.5 \sigma$-clipped mean $E(B-V)$ value and we used the equations reported by Stassun et al. (2019) to correct the colours and the magnitudes of the stars.

We selected the main sequence stars as follows: first, we excluded the evolved stars of each open cluster visually inspecting each CMD. In a second step, we determined the fiducial line of all the main sequence stars by using the naive estimator (Silverman 1986, see Nardiello et al. 2015b for a detailed description of the method), and we selected all the stars whose colours are within $2 \sigma$ from the mean colour of the fiducial line. Green, blue, and magenta points in Fig. 9 are the main sequence stars with radii $R_{\star} \lesssim 0.5 R_{\odot}$, $0.5 R_{\odot} \lesssim R_{\star} \lesssim 1.0 R_{\odot}$ and $1.0 R_{\odot} \lesssim R_{\star} \lesssim 1.5 R_{\odot}$, respectively, and that will be used in the following analysis.

The three panels of Fig. 8 show that we are not able to detect transiting exoplanets with $R_{\mathrm{P}} \leq 1 R_{\mathrm{E}}$ around cluster members with $R_{\star} \gtrsim 0.5 R_{\odot}$ : indeed, in order to be able to detect this kind of exoplanets it would be necessary that the hosting star is at a distance $d \lesssim 30 \mathrm{pc}$, but no open clusters studied in this work satisfies this condition. In the case of super-Earths $\left(R_{\mathrm{P}} \lesssim 2 R_{\mathrm{E}}\right)$, we have no possibility to find them around cluster members with $R_{\star}<0.5 R_{\odot}$, but 


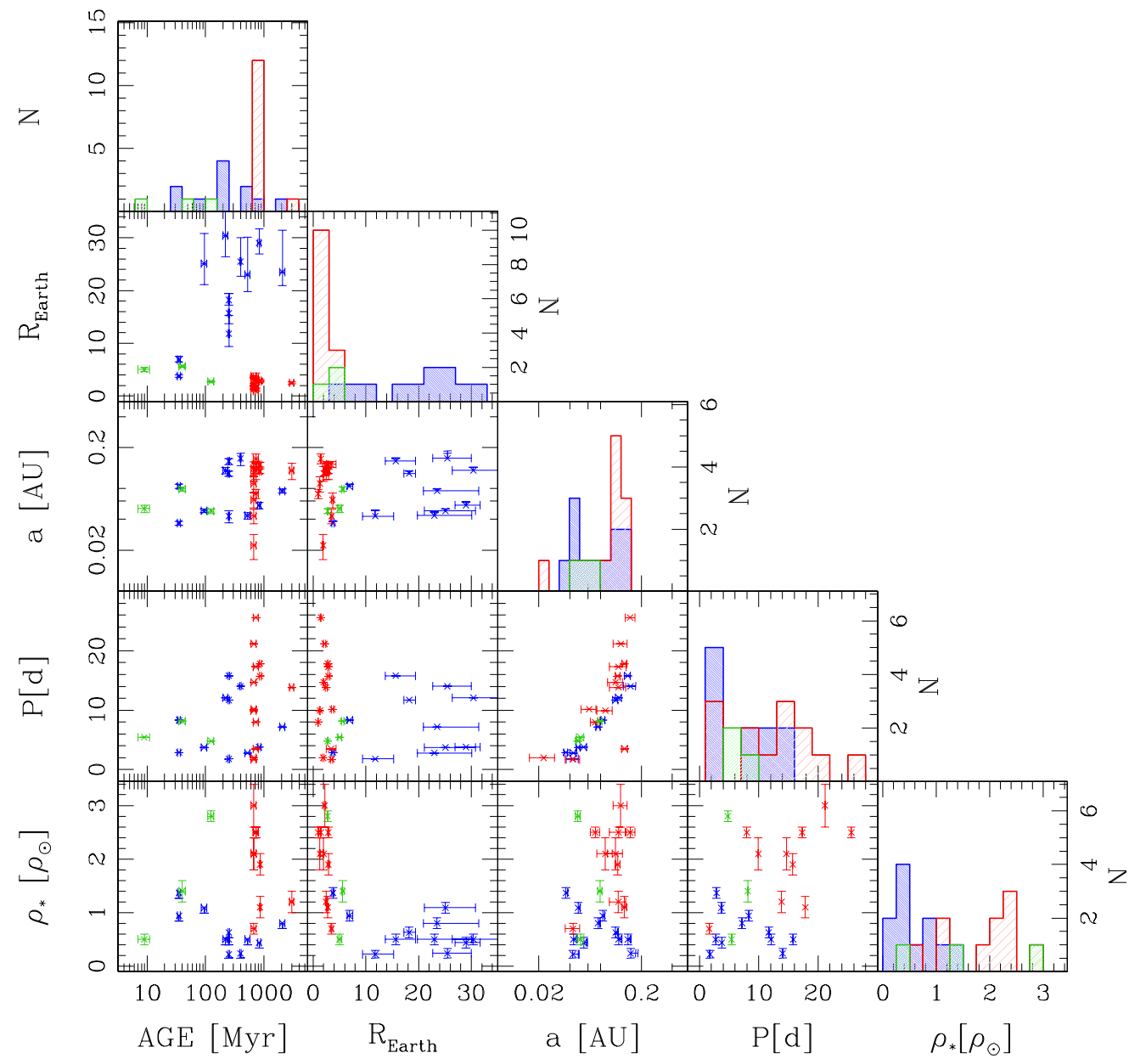

Figure 7. Correlations between cluster, stellar, and exoplanets properties. Blue points and histograms are the results of this work; red points and histograms are the results obtained using data of open clusters collected by Kepler; green points and histograms relative to stars in associations.

we are able to detect this kind of exoplanets in the light curves of stars with $0.5 R_{\odot} \lesssim R_{\star} \lesssim 1.5 R_{\odot}$ and located at $d \lesssim 150$ pc. For the two intervals of stellar radii $0.5 R_{\odot} \lesssim$ $R_{\star} \lesssim 1.0 R_{\odot}$ and $1.0 R_{\odot} \lesssim R_{\star} \lesssim 1.5 R_{\odot}$, from the $\mathrm{CMD}$ of Fig. 9 combined with information on the distance from Fig. 8, we extracted a total of $N_{\star}=23 \pm 5$ and $N_{\star}=20 \pm 4$ stars with these radii, respectively. In our survey we detected no super-Earth transits. This result is in agreement with the frequency of field exoplanets from the Kepler survey, as tabulated by Fressin et al. (2013).

Following Curtis et al. (2018), we also calculated the number of expected transiting super-Earths in our sample, by using the equation

$N_{\text {planet }}=N_{\star} \times f_{\star} \times \operatorname{Pr}_{\text {transit }}$

where $f_{\star}=4.82 \%$ is the percentage of stars with at least one exoplanet for the period range 0.8-10 d estimated by Fressin et al. (2013), $\operatorname{Pr}_{\text {transit }} \simeq R_{\star} / a$ is the transit probability, and $a$ is calculated assuming an average period $P=10 \mathrm{~d}$. The result of this estimate is $N_{\text {planet }} \simeq 0$ for stars with $R_{\star}=1.0 R_{\odot}$ and $R_{\star}=1.5 R_{\odot}$, consistent with our null detection.

From Figs. 8 and 9, transiting exoplanets having a radius $R_{\mathrm{P}} \sim 1 R_{\mathrm{N}}$ and orbiting stars with $R_{\star} \lesssim 0.5 R_{\odot}$ can be detected in the light curve of $N_{\star}=23 \pm 5$ stars. Fressin et al. (2013) estimated that $f_{\star}=2.61 \%$ of field stars host Neptune-size exoplanets with periods $<10 \mathrm{~d}$. Correspondingly, we expect a null detection (0.02 planets) of Neptunesize exoplanets transiting low mass main sequence stars that are members of the studied clusters.

In the case the hosting star has radius $0.5 R_{\odot} \lesssim R_{\star} \lesssim 1.0 R_{\odot}$ and $1.0 R_{\odot} \lesssim R_{\star} \lesssim 1.5 R_{\odot}$, we are able to detect Neptunesize transits in the light curves of $N_{\star}=1086 \pm 33$ and $N_{\star}=1868 \pm 43$ stars. We found 1 Neptune-size exoplanet around a $R_{\star} \sim 1.1 R_{\odot}$ star (PATHOS-30), while PATHOS-31 is a candidate Neptune orbiting a $R_{\star} \sim 0.8 R_{\odot}$ star. By using these information, we calculated the frequency of transiting Neptune exoplanets for stars with $0.5 R_{\odot} \lesssim R_{\star} \lesssim 1.5 R_{\odot}$, $f_{\star}$, from the equation (2). We found that for $0.5 R_{\odot} \lesssim R_{\star} \lesssim$ $1.0 R_{\odot}, f_{\star}=1.84 \pm 1.84 \%$, while for $1.0 R_{\odot} \lesssim R_{\star} \lesssim 1.5 R_{\odot}$ we obtained $f_{\star}=0.67 \pm 0.67 \%$; if we consider all the stars with $R \lesssim 1.5 R_{\odot}$ we have a frequency of transiting Neptunes $f_{\star}=1.34 \pm 0.95 \%$, in agreement with Fressin et al. (2013), who found. $f_{\star}=2.61 \pm 0.18 \%$, within $\sim 1 \sigma$.

In our survey, Jupiter-size exoplanets orbiting stars of radius $R_{\star} \lesssim 0.5 R_{\odot}$ can be detected in the light curves 

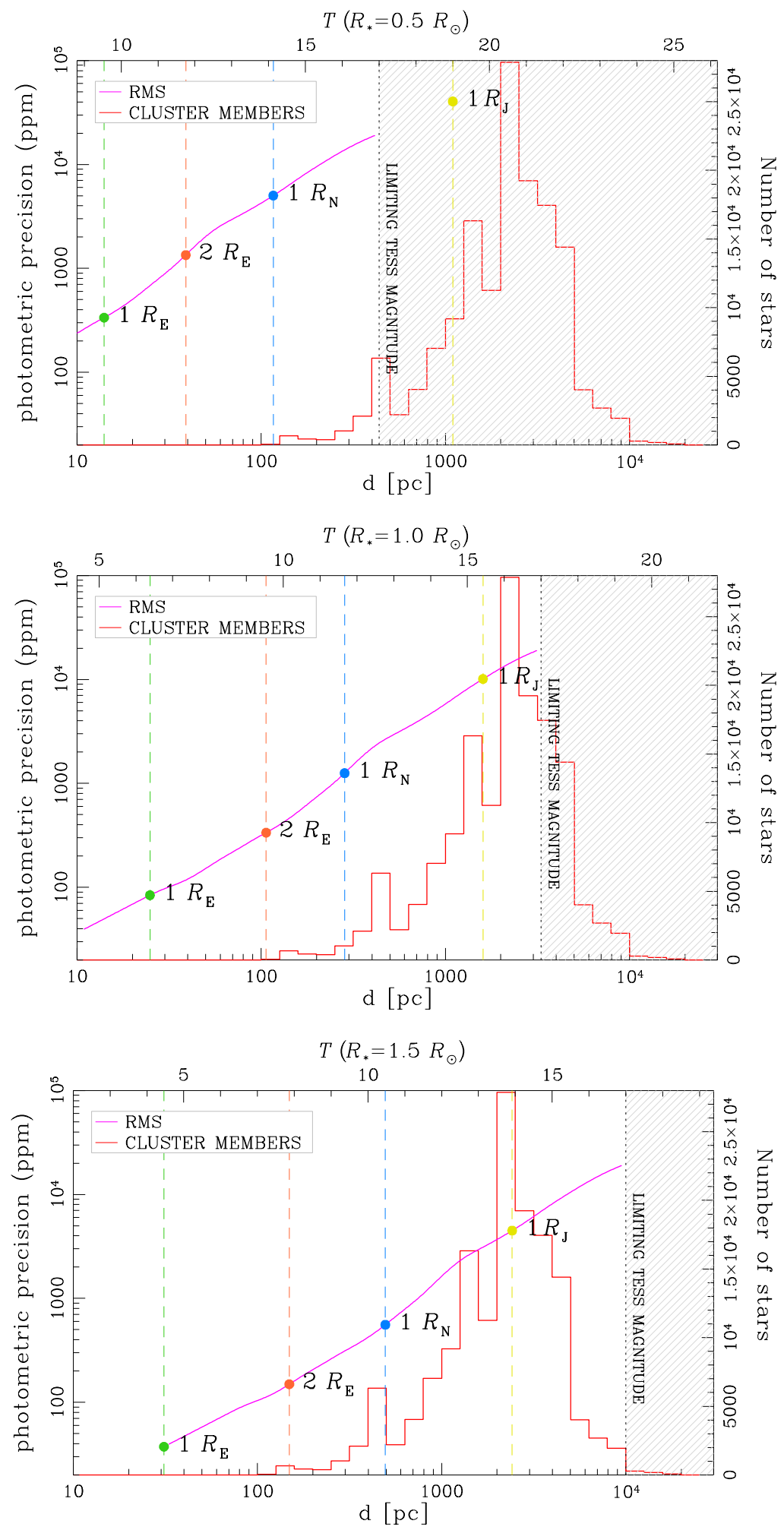

Figure 8. Detectability limits of transits of exoplanets of size $R_{P}=1 R_{\mathrm{E}}$ (green dot), $R_{P}=2 R_{\mathrm{E}}$ (orange dot), $R_{P}=1 R_{\mathrm{N}}$ (light blue dot), and $R_{P}=1 R_{\mathrm{J}}$ (yellow dot) orbiting stars with radius $R_{\star}=0.5 R_{\odot}$ (top panel), $R_{\star}=1.0 R_{\odot}\left(\right.$ middle panel), and $R_{\star}=1.5 R_{\odot}$ (bottom panel). Magenta line represents the average RMS distribution as a function of the magnitude obtained from flattened light curves. Red histogram is the distance distribution of the cluster members studied in this work. Grey zone represents the range of magnitudes outside the range of detection of TESS. The upper axis of each panel gives the apparent magnitude of a star with $R_{\star}=X R_{\odot}$, with $X=0.5,1.0,1.5$, at the corresponding distance of the lower axis. 


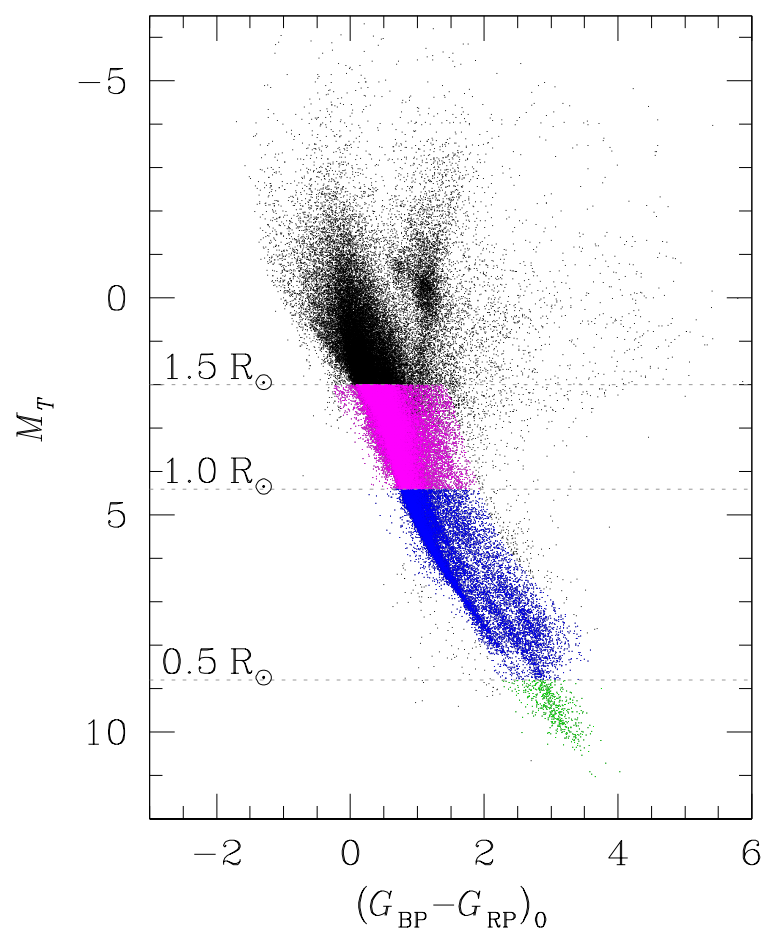

Figure 9. The $M_{T}$ versus $\left(G_{\mathrm{BP}}-G_{\mathrm{RP}}\right)_{0}$ CMD for the analysed stars in this work. Green, blue, and magenta points are the main sequence stars in the radii intervals $R_{\star} \lesssim 0.5 R_{\odot}, 0.5 R_{\odot} \lesssim R_{\star} \lesssim$ $1.0 R_{\odot}$ and $1.0 R_{\odot} \lesssim R_{\star} \lesssim 1.5 R_{\odot}$, respectively.

of $N_{\star}=544 \pm 23$ cluster members, while, for stars with $0.5 R_{\odot} \lesssim R_{\star} \lesssim 1.0 R_{\odot}$ and $1.0 R_{\odot} \lesssim R_{\star} \lesssim 1.5 R_{\odot}$, we were able to detect transits of this kind of exoplanets in the light curves of $N_{\star}=17583 \pm 133$ and $N_{\star}=26776 \pm 164$ cluster stars, respectively. We did not detect any Jupitersize exoplanet around stars with $R_{\star} \lesssim 0.5 R_{\odot}$; it is expected assuming a frequency of giant planets $(\sim 0.43 \%)$ as in Fressin et al. (2013). We calculated the observed frequency of transiting Jupiters in the other two ranges of stellar radii, following the procedure already adopted for Neptune-size planets. We found 1 giant exoplanets orbiting $0.5 R_{\odot} \lesssim R_{\star} \lesssim 1.0 R_{\odot}$, with a corresponding frequency $f_{\star}=0.11 \pm 0.11 \% ; 6$ Jupiter-size exoplanets were detected for stars with radii $1.0 R_{\odot} \lesssim R_{\star} \lesssim 1.5 R_{\odot}$, with a frequency of $f_{\star}=0.28 \pm 0.11 \%$. Considering all the stars with $R_{\star} \lesssim 1.5 R_{\odot}$ for which we are able to detect Jupiter-size transiting exoplanets, we obtain a frequency $f_{\star}=0.19 \pm 0.07 \%$, that is significantly lower than that found by Fressin et al. (2013, $\left.f_{\star}=(0.43 \pm 0.05) \%\right)$.

We want to emphasise that, in the statistical analysis we performed, there two effects that partially compensate each other and that can affect our estimated frequencies: (i) our detection method might miss some transit, i.e., the sample of detected candidate exoplanets might be not complete. Including the completeness correction might result in a higher value of $f_{\star}$; (ii) though we applied a validation check to our targets, we could not identify all false positives, estimated to be $\sim 40 \%$ by Sullivan et al. (2015), and $\sim 33 \%$ by Cloutier (2019). Because of our false positive check, the fraction of false positives in our sample is surely smaller, but not null, and this fact would decrease $f_{\star}$.

\section{SUMMARY AND CONCLUSION}

In the second work of our project PATHOS, we applied our PSF-based approach to TESS FFIs in order to extract highprecision light curves of stars that belong to open clusters observed by TESS during the first year of its mission (Sectors 1-13). We extracted and analysed 219256 light curves of 162901 stars located in 645 open clusters ( 51475 stars are observed in more than one TESS sector). These open clusters span a wide range of ages (from few tens Myr up to $\sim 3 \mathrm{Gyr}$ ) and distances $\left(\sim 100-10^{4} \mathrm{pc}\right)$, allowing us to probe stars born at different times and in different environmental conditions. The light curves will be publicly available as HLSP on the PATHOS project webpage ${ }^{8}$ (DOI: $10.17909 /$ t9-es7mvw14) of the MAST archive.

We searched for transit signals among the extracted and corrected light curves and, after a series of vetting tests, we isolated 33 transiting objects of interest. We extracted the physical parameters of these objects modelling the transits, and using these information combined with the stellar properties, we selected 11 candidate exoplanets orbiting stars of 8 open clusters. One of the youngest open clusters in our sample, IC 2602 ( $35 \mathrm{Myr}$ ), hosts two stars with candidate Neptune-size exoplanets; while in the $250 \mathrm{Myr}$ old open cluster NGC 2516, we found two warm and one hot Jupiter candidates. In the remaining clusters were we detected one transiting candidate exoplanet in each of them: NGC 2112 ( 2 Gyr $),$ NGC 2323 ( 100 Myr $)$, NGC 2437 ( $220 \mathrm{Myr})$, NGC $2527(\sim 830 \mathrm{Myr})$, NGC $2548(\sim 500 \mathrm{Myr})$, and NGC $3532(\sim 400 \mathrm{Myr})$

We also show that the planet detection is strongly affected by the bias due to the distance of the open clusters. Comparing the mean distribution of the photometric precision with the expected depth of the transits due to exoplanets with different radii and the percentage of stars that host different size exoplanets tabulated by Fressin et al. (2013), we expected a null detection of Earth and super-Earth size exoplanets in the light curves of the members of the open clusters analysed in this work.

We detected 2 Neptune-size candidate exoplanets around two IC 2602 members with radii $R_{\star} \lesssim 1.5 R_{\odot}$; consequently, we estimated that the frequency of this kind of exoplanets is $f_{\star}=1.34 \pm 0.95$, consistent with that of field stars.

We also identified 7 Jupiters around stars with $R_{\star} \lesssim$ $1.5 R_{\odot}$ and estimated a fraction $f_{\star}=0.19 \pm 0.07 \%$ for this kind of planets, significantly smaller than what estimated for field stars (e.g., Fressin et al. 2013). Two, partially compensating effects (completeness of our detection method and false positive rate), make our results still provisional.

The analysis of the light curves of open cluster members in the northern ecliptic hemisphere, as also the analysis of cluster members that will be observed during the TESS extended mission(s), will be important for a better estimate of planet fraction in cluster stars. Spectroscopic follow-up, when feasible, is mandatory as well, in order to confirm the planetary nature and derive the exoplanet masses and search for a correlation between planet composition and cluster properties.

8 https://archive.stsci.edu/hlsp/pathos 


\section{ACKNOWLEDGEMENTS}

DN acknowledges support from the French Centre National d'Etudes Spatiales (CNES). DN and GP recognize partial support by UNIPD/DFA Dipartimental project PIOT_SID17_01. GP, VG, LM, MM, DN, acknowledge support from PLATO ASI-INAF agreements n.2015-019-R02015 and n. 2015-019-R.1-2018. LB, GP, DN acknowledge the funding support from CHEOPS ASI-INAF agreement $n$. 2019-29-HH.0. LRB acknowledges support by MIUR under PRIN program \#2017Z2HSMF. The authors warmly thank the anonymous referee for the prompt and careful reading of our manuscript.

\section{REFERENCES}

Alard C., Lupton R. H., 1998, ApJ, 503, 325

Anderson J., et al., 2008, AJ, 135, 2055

Bailer-Jones C. A. L., Rybizki J., Fouesneau M., Mantelet G., Andrae R., 2018, AJ, 156, 58

Bailey J. I., Mateo M., White R. J., Shectman S. A., Crane J. D., 2018, MNRAS, 475, 1609

Barros S. C. C., Demangeon O., Deleuil M., 2016, A\&A, 594, A100

Bellini A., Anderson J., Bedin L. R., King I. R., van der Marel R. P., Piotto G., Cool A., 2017, ApJ, 842, 6

Benatti S., et al., 2019, A\&A, 630, A81

Bica E., Bonatto C., 2011, A\&A, 530, A32

Binney J., et al., 2014, MNRAS, 437, 351

Borucki W. J., et al., 2010, Science, 327, 977

Bossini D., et al., 2019, A\&A, 623, A108

Bouma L. G., Hartman J. D., Bhatti W., Winn J. N., Bakos G. Á., 2019, ApJS, 245, 13

Brucalassi A., et al., 2014, A\&A, 561, L9

Brucalassi A., et al., 2016, A\&A, 592, L1

Brucalassi A., et al., 2017, A\&A, 603, A85

Cantat-Gaudin T., et al., 2018, A\&A, 618, A93

Chang S. W., Byun Y. I., Hartman J. D., 2015, AJ, 149, 135

Ciardi D. R., et al., 2018, AJ, 155, 10

Claret A., 2018, A\&A, 618, A20

Cloutier R., 2019, AJ, 158, 81

Curtis J. L., et al., 2018, AJ, 155, 173

David T. J., et al., 2016a, AJ, 151, 112

David T. J., et al., 2016b, Nature, 534, 658

David T. J., et al., 2018, AJ, 156, 302

Foreman-Mackey D., Hogg D. W., Lang D., Goodman J., 2013, PASP, 125, 306

Fressin F., et al., 2013, ApJ, 766, 81

Gaia Collaboration et al., 2018, A\&A, 616, A1

Gaidos E., et al., 2017, MNRAS, 464, 850

Gaidos E., et al., 2020, arXiv e-prints, p. arXiv:2003.12940

Gilliland R. L., 2004, Technical report, ACS CCD Gains, Full Well Depths, and Linearity up to and Beyond Saturation

Gilliland R. L., Rajan A., Deustua S., 2010, Technical report, WFC3 UVIS Full Well Depths, and Linearity Near and Beyond Saturation

Hidalgo S. L., et al., 2018, ApJ, 856, 125

Hippke M., Heller R., 2019, A\&A, 623, A39

Howell S. B., et al., 2014, PASP, 126, 398

Kharchenko N. V., Piskunov A. E., Schilbach E., Röser S., Scholz R. D., 2016, A\&A, 585, A101

Kipping D. M., 2010, MNRAS, 408, 1758

Kipping D. M., 2013, MNRAS, 435, 2152

Kreidberg L., 2015, PASP, 127, 1161

Lallement R., et al., 2018, A\&A, 616, A132
Libralato M., Bedin L. R., Nardiello D., Piotto G., 2016a, MNRAS, 456, 1137

Libralato M., et al., 2016b, MNRAS, 463, 1780

Malavolta L., et al., 2016, A\&A, 588, A118

Malavolta L., et al., 2018, AJ, 155, 107

Mann A. W., et al., 2016a, AJ, 152, 61

Mann A. W., et al., 2016b, ApJ, 818, 46

Mann A. W., et al., 2017, AJ, 153, 64

Mann A. W., et al., 2018, AJ, 155, 4

Meibom S., et al., 2013, Nature, 499, 55

Moré J. J., Garbow B. S., Hillstrom K. E., 1980, Technical Report ANL-80-74, User guide for MINPACK-1. Argonne Nat. Lab., Argonne, IL

Nardiello D., et al., 2015a, MNRAS, 447, 3536

Nardiello D., et al., 2015b, MNRAS, 451, 312

Nardiello D., Libralato M., Bedin L. R., Piotto G., Ochner P., Cunial A., Borsato L., Granata V., 2016a, MNRAS, 455, 2337

Nardiello D., Libralato M., Bedin L. R., Piotto G., Borsato L., Granata V., Malavolta L., Nascimbeni V., 2016b, MNRAS, 463,1831

Nardiello D., et al., 2018, MNRAS, 481, 3382

Nardiello D., et al., 2019, MNRAS, 490, 3806

Netopil M., Paunzen E., Heiter U., Soubiran C., 2016, A\&A, 585, A150

Newton E. R., et al., 2019, ApJ, 880, L17

Obermeier C., et al., 2016, AJ, 152, 223

Pepper J., et al., 2017, AJ, 153, 177

Pope B. J. S., Parviainen H., Aigrain S., 2016, MNRAS, 461, 3399

Quinn S. N., et al., 2012, ApJ, 756, L33

Quinn S. N., et al., 2014, ApJ, 787, 27

Ricker G. R., et al., 2015, Journal of Astronomical Telescopes, Instruments, and Systems, 1, 014003

Sato B., et al., 2007, ApJ, 661, 527

Silverman B. W., 1986, Density estimation for statistics and data analysis

Soares-Furtado M., Hartman J. D., Bakos G. Á., Huang C. X., Penev K., Bhatti W., 2017, PASP, 129, 044501

Soares-Furtado M., Hartman J. D., Bhatti W., Bouma L. G., Barna T., Bakos G. Á., 2020, ApJS, 246, 15

Stassun K. G., et al., 2019, AJ, 158, 138

Sullivan P. W., et al., 2015, ApJ, 809, 77

Vanderburg A., et al., 2018, AJ, 156, 46

Wallace J. J., Hartman J. D., Bakos G. Á., Bhatti W., 2019, ApJS, 244, 12

\section{APPENDIX A: LIGHT CURVE MODELLING AND EXOPLANET PARAMETERS ESTIMATE}

This paper has been typeset from a $\mathrm{T}_{\mathrm{E} X} \mathrm{~L} \mathrm{LT}_{\mathrm{E}} \mathrm{X}$ file prepared by the author. 

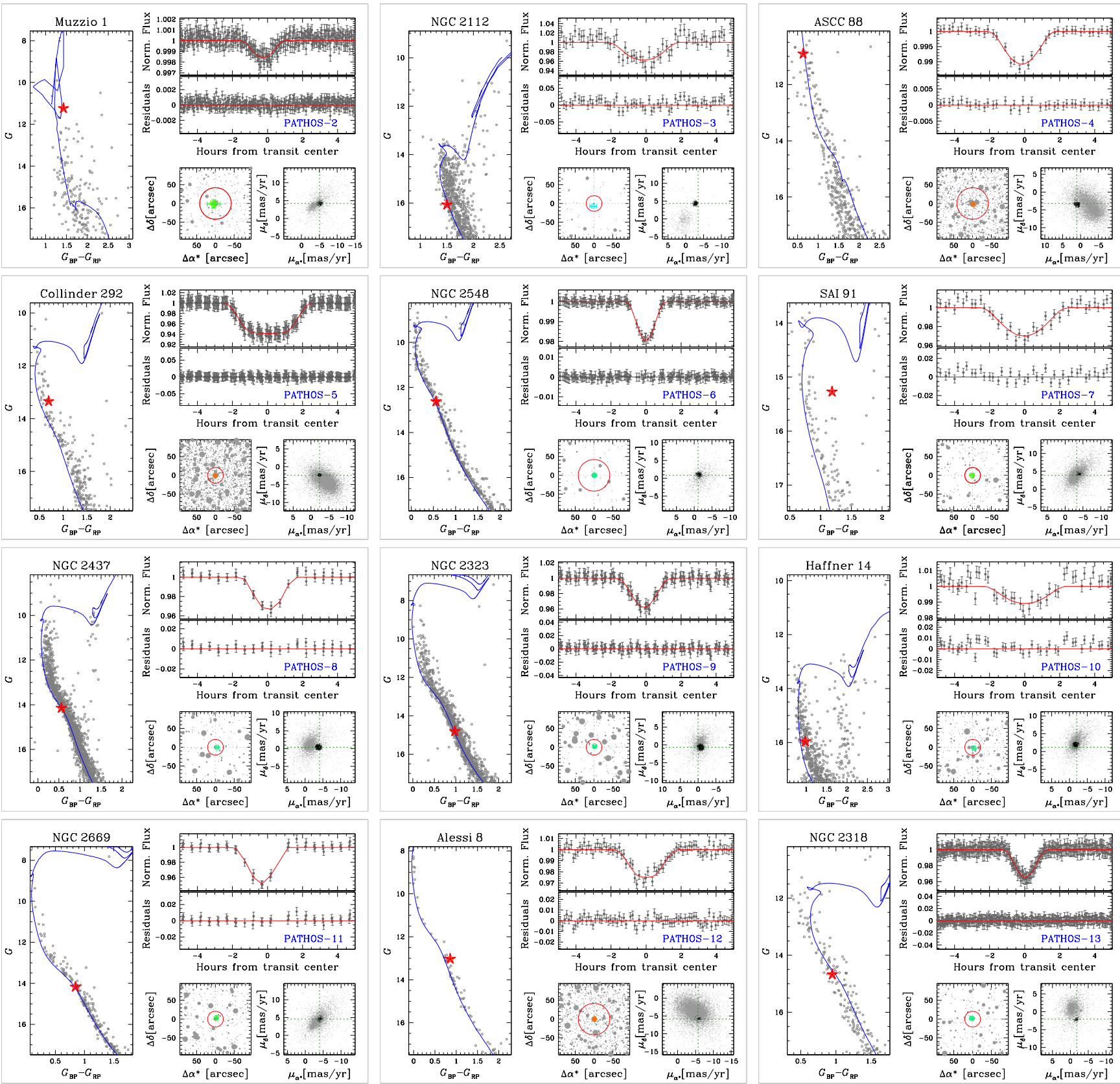

Figure A1. Overview on the candidate exoplanets PATHOS-2 - PATHOS-13. On the left-hand, the $G$ versus $G_{\mathrm{BP}}-G_{\mathrm{RP}}$ CMD of the cluster that hosts the target star (red star) and the isochrone (blue) fitted with the cluster parameters listed in Table 1. On the right-hand, top-panel shows the folded light curve (grey points) of the candidate and the model (in red) found with PyORBIT; middle panel shows the difference between the observed points and the model. Bottom left-hand panel shows the $95 \times 95 \operatorname{arcsec}^{2}$ finding chart centred on the target star; red circle is the aperture adopted to extract photometry, crosses are the in-/out-of transit difference centroid, colour-coded as in Fig. 6. Bottom-right panel is the vector-point diagram, centred on the target star, for all the stars that are within 10 arcmin from the target star; black points are the cluster members listed in the catalogue by Cantat-Gaudin et al. (2018). 

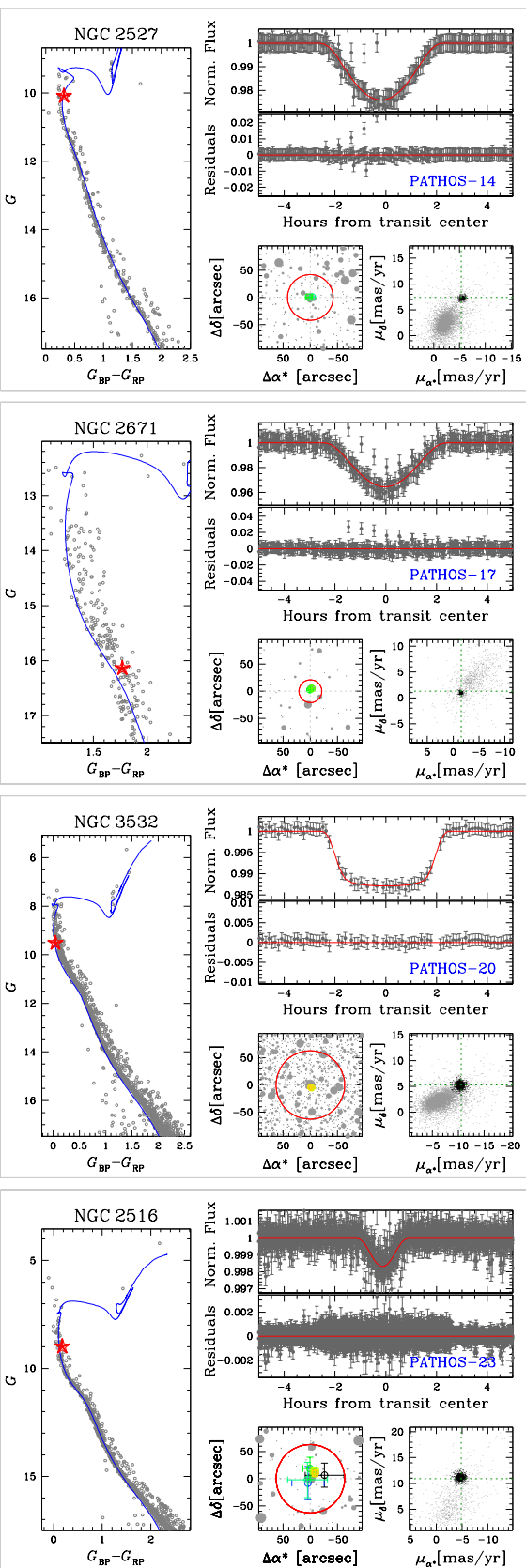
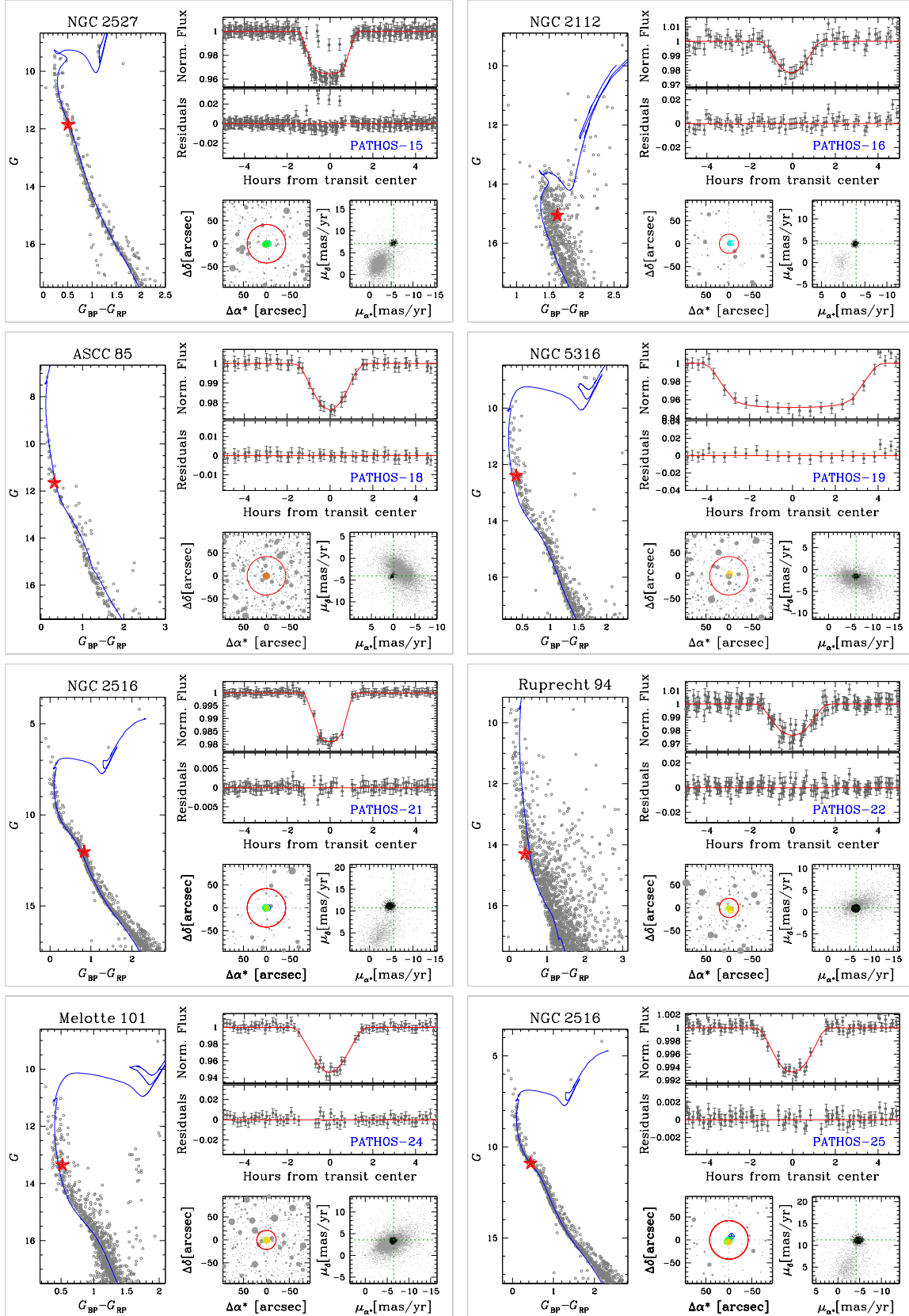
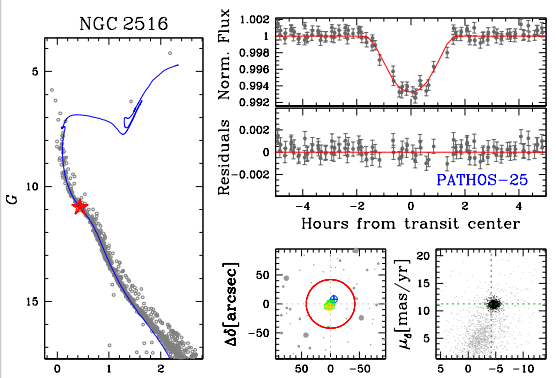

Figure A2. As in Fig. A1, but for PATHOS-14 - PATHOS-25. 

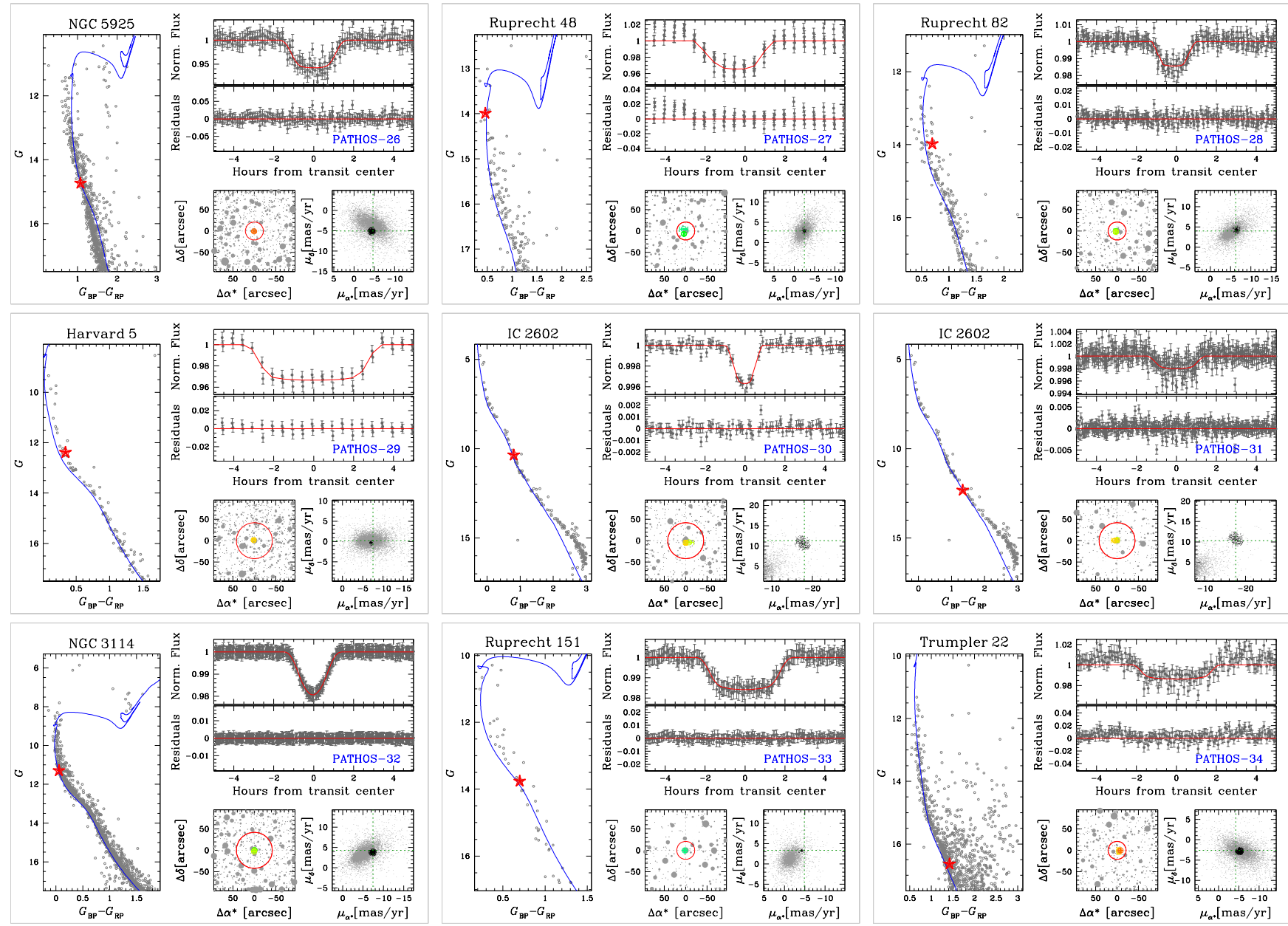

Figure A3. As in Fig. A1, but for PATHOS-26 - PATHOS-34. 
Table A1. Results of transit modelling

\begin{tabular}{|c|c|c|c|c|c|c|c|c|c|c|c|c|c|c|}
\hline TIC & PATHOS & Cluster & $\begin{array}{l}P \\
\text { (d) }\end{array}$ & $\begin{array}{c}T_{0} \\
\text { (BTJD) }\end{array}$ & $R_{\mathrm{p}} / R_{\star}$ & $b$ & $a / R_{\star}$ & $\begin{array}{l}\rho_{\star} \\
\left(\rho_{\odot}\right)\end{array}$ & $\mathrm{LD}_{c 1}$ & $\mathrm{LD}_{c 2}$ & $\begin{array}{l}i \\
(\operatorname{deg})\end{array}$ & $\begin{array}{l}R_{\mathrm{p}} \\
\left(R_{\mathrm{J}}\right)\end{array}$ & $\begin{array}{l}R_{\mathrm{p}} \\
\left(R_{\oplus}\right)\end{array}$ & Note \\
\hline 0030654608 & 2 & Muzzio 1 & $2.4955_{-0.0003}^{+0.0003}$ & $1519.662_{-0.009}^{+0.009}$ & $0.048_{-0.009}^{+0.014}$ & $0.96_{-0.16}^{+0.03}$ & $3.7_{-0.6}^{+0.6}$ & $0.11_{-0.04}^{+0.06}$ & $0.38_{-0.26}^{+0.35}$ & $0.18_{-0.28}^{+0.32}$ & $71.7_{-13.6}^{+5.2}$ & $2.5_{-0.7}^{+1.0}$ & $28.6_{-8.0}^{+10.8}$ & \\
\hline 0039291805 & 3 & NGC 2112 & $7.1719_{-0.0059}^{+0.0057}$ & $1469.033_{-0.009}^{+0.0099}$ & $0.190_{-0.018}^{+0.004}$ & $0.69_{-0.40}^{+0.21}$ & $14.5_{-0.6}^{+0.6}$ & $0.80_{-0.10}^{+0.10}$ & $0.31_{-0.05}^{+0.05}$ & $0.29_{-0.05}^{+0.05}$ & $87.1_{-0.8}^{+1.2}$ & $2.1_{-0.2}^{+0.7}$ & $23.5_{-2.6}^{+7.9}$ & \\
\hline 0042524156 & 4 & ASCC 88 & $10.0130_{-0.0026}^{+0.0025}$ & $1631.474_{-0.006}^{+0.005}$ & $0.125_{-0.019}^{+0.024}$ & $0.94_{-0.06}^{+0.04}$ & $12.0_{-1.8}^{+1.4}$ & $0.23_{-0.09}^{+0.09}$ & $0.18_{-0.11}^{+0.12}$ & $0.16_{-0.12}^{+0.13}$ & $84.0_{-3.8}^{+1.6}$ & $3.5_{-0.7}^{+0.8}$ & $39.1_{-7.3}^{+9.5}$ & \\
\hline 0080317933 & 5 & Collinder 292 & $3.2098_{-0.0006}^{+0.0006}$ & $1625.529_{-0.003}^{+0.003}$ & $0.232_{-0.005}^{+0.006}$ & $0.39_{-0.24}^{+0.17}$ & $6.3_{-0.6}^{+0.5}$ & $0.32_{-0.09}^{+0.09}$ & $0.19_{-0.05}^{+0.05}$ & $0.23_{-0.05}^{+0.05}$ & $86.3_{-1.8}^{+2.2}$ & $4.1_{-0.3}^{+0.5}$ & $45.8_{-3.8}^{+5.4}$ & \\
\hline 0088977253 & 6 & NGC 2548 & $2.7677_{-0.0004}^{+0.0004}$ & $1492.708_{-0.004}^{+0.004}$ & $0.146_{-0.016}^{+0.048}$ & $0.85_{-0.31}^{+0.11}$ & $6.6_{-0.5}^{+0.4}$ & $0.50_{-0.10}^{+0.10}$ & $0.27_{-0.05}^{+0.05}$ & $0.28_{-0.05}^{+0.05}$ & $78.1_{-4.3}^{+2.7}$ & $2.1_{-0.3}^{+0.6}$ & $23.0_{-3.2}^{+7.1}$ & TOI-496 \\
\hline 0092835691 & 7 & SAI 91 & $22.3661_{-0.0069}^{+0.0067}$ & $1527.825_{-0.007}^{+0.007}$ & $0.214_{-0.033}^{+0.0034}$ & $0.92_{-0.07}^{+0.05}$ & $20.0_{-3.1}^{+2.5}$ & $0.22_{-0.09}^{+0.09}$ & $0.19_{-0.05}^{+0.05}$ & $0.23_{-0.05}^{+0.05}$ & $85.4_{-2.9}^{+1.3}$ & $4.4_{-0.8}^{+1.0}$ & $49.8_{-9.3}^{+11.2}$ & \\
\hline 0094589619 & 8 & NGC 2437 & $12.0829_{-0.0047}^{+0.0045}$ & $1496.198_{-0.004}^{+0.004}$ & $0.187_{-0.019}^{+0.062}$ & $0.81_{-0.39}^{+0.14}$ & $17.6_{-1.2}^{+1.1}$ & $0.50_{-0.10}^{+0.10}$ & $0.24_{-0.05}^{+0.05}$ & $0.26_{-0.05}^{+0.05}$ & $84.9_{-1.9}^{+1.3}$ & $2.7_{-0.4}^{+0.8}$ & $30.4_{-4.0}^{+9.4}$ & \\
\hline 0125414447 & 9 & NGC 2323 & $3.7069_{-0.0008}^{+0.00008}$ & $1493.200_{-0.004}^{+0.004}$ & $0.229_{-0.035}^{+0.052}$ & $0.88_{-0.10}^{+0.09}$ & $10.4_{-0.3}^{+0.3}$ & $1.09_{-0.10}^{+0.10}$ & $0.32_{-0.05}^{+0.05}$ & $0.30_{-0.05}^{+0.05}$ & $85.4_{-0.8}^{+0.5}$ & $2.2_{-0.4}^{+0.5}$ & $25.1_{-4.0}^{+5.7}$ & \\
\hline 0126600730 & 10 & Haffner 14 & $6.2991_{-0.0067}^{+0.0067}$ & $1497.305_{-0.009}^{+0.011}$ & $0.102_{-0.010}^{+0.027}$ & $0.75_{-0.46}^{+0.18}$ & $9.3_{-1.2}^{+1.0}$ & $0.27_{-0.09}^{+0.10}$ & $0.15_{-0.05}^{+0.05}$ & $0.20_{-0.05}^{+0.05}$ & $84.7_{-2.4}^{+1.9}$ & $2.1_{-0.3}^{+0.7}$ & $23.8_{-3.7}^{+8.0}$ & \\
\hline 0144995073 & 11 & NGC 2669 & $20.0207_{-0.0050}^{+0.0051}$ & $1518.823_{-0.005}^{+0.005}$ & $0.240_{-0.038}^{+0.066}$ & $0.83_{-0.34}^{+0.12}$ & $25.6_{-1.6}^{+1.4}$ & $0.56_{-0.10}^{+0.10}$ & $0.27_{-0.05}^{+0.05}$ & $0.28_{-0.05}^{+0.05}$ & $84.9_{-2.3}^{+1.4}$ & $3.2_{-0.5}^{+0.8}$ & $36.0_{-6.1}^{+9.5}$ & \\
\hline 0147069011 & 12 & Alessi 8 & $\begin{array}{r}7.3270_{-0.0018}^{+0.0019}\end{array}$ & $1628.559_{-0.004}^{+0.004}$ & $0.448_{-0.045}^{+0.035}$ & $0.38_{-0.25}^{+0.22}$ & $13.9_{-0.7}^{+0.6}$ & $0.67_{-0.10}^{+0.10}$ & $0.28_{-0.05}^{+0.05}$ & $0.29_{-0.05}^{+0.05}$ & $86.8_{-1.6}^{+1.9}$ & $5.4_{-0.6}^{+0.5}$ & $61.0_{-6.5}^{+5.6}$ & \\
\hline 0147426828 & 13 & NGC 2318 & $1.4618_{-0.0002}^{+0.0002}$ & $1492.845_{-0.004}^{+0.004}$ & $0.221_{-0.037}^{+0.047}$ & $0.89_{-0.12}^{+0.08}$ & $4.2_{-0.3}^{+0.3}$ & $0.47_{-0.09}^{+0.09}$ & $0.25_{-0.05}^{+0.05}$ & $0.27_{-0.05}^{+0.05}$ & $76.2_{-3.9}^{+2.2}$ & $3.2_{-0.6}^{+0.7}$ & $35.9_{-6.4}^{+7.6}$ & \\
\hline 0153734545 & 14 & NGC 2527 & $3.2196_{-0.0003}^{+0.0003}$ & $1493.665_{-0.008}^{+0.008}$ & $0.187_{-0.025}^{+0.034}$ & $.92_{-0.06}^{+0.06}$ & $4.5_{-0.6}^{+0.8}$ & $0.11_{-0.04}^{+0.07}$ & $0.22_{-0.05}^{+0.05}$ & $0.25_{-0.05}^{+0.05}$ & $78.1_{-5.1}^{+3.1}$ & $4.8_{-0.9}^{+1.0}$ & $53.7_{-10.4}^{+13.4}$ & \\
\hline 0153735144 & 15 & NGC 2527 & $3.7785_{-0.0003}^{+0.0003}$ & $1493.406_{-0.002}^{+0.0002}$ & $0.178_{-0.004}^{+0.006}$ & $0.37_{-0.25}^{+0.24}$ & $7.8_{-0.6}^{+0.5}$ & $0.44_{-0.10}^{+0.10}$ & $0.26_{-0.05}^{+0.05}$ & $0.27_{-0.05}^{+0.05}$ & $84.4_{-2.6}^{+3.5}$ & $2.6_{-0.2}^{+0.2}$ & $29.0_{-2.1}^{+2.7}$ & \\
\hline 0159059181 & 16 & NGC 2112 & $5.2167_{-0.0020}^{+0.00030}$ & $1471.198_{-0.005}^{+0.0005}$ & $0.162_{-0.025}^{+0.0048}$ & $\begin{array}{r}0.87_{-0.31}^{-0.09} \\
0.09\end{array}$ & $9.3_{-0.8}^{+0.0}$ & $0.40_{-0.09}^{+0.10}$ & $\begin{array}{r}0.28_{-0.05}^{+0.05} \\
0.05\end{array}$ & $0.28_{-0.05}^{-0.05}$ & $\begin{array}{r}-2.0 \\
82.5_{-3.3}^{+1.8}\end{array}$ & $2.4_{-0.4}^{+0.2}$ & $27.0_{-4.8}^{+7.1}$ & \\
\hline 0181602717 & 17 & NGC 2671 & $2.4557_{-0.0002}^{+0.0002}$ & $1517.489_{-0.009}^{+0.009}$ & $0.246_{-0.034}^{+0.030}$ & $.93_{-0.05}^{+0.05}$ & $5.9_{-0.5}^{+0.4}$ & $0.46_{-0.10}^{+0.10}$ & $0.25_{-0.05}^{+0.05}$ & $0.25_{-0.05}^{+0.05}$ & $83.2_{-1.5}^{+0.9}$ & $3.6_{-0.5}^{-0.4}$ & $40.2_{-5.8}^{+6.8}$ & \\
\hline 0236084210 & 18 & ASCC 85 & $5.3465_{-0.0016}^{+0.0016}$ & $1631.974_{-0.004}^{+0.004}$ & $0.182_{-0.028}^{+0.035}$ & $0.91_{-0.07}^{+0.06}$ & $9.8_{-0.8}^{+0.6}$ & $0.44_{-0.09}^{+0.09}$ & $0.15_{-0.05}^{+0.05}$ & $0.18_{-0.05}^{+0.05}$ & $83.9_{-1.8}^{+0.9}$ & $3.3_{-0.5}^{+0.6}$ & $36.6_{-5.8}^{+7.2}$ & \\
\hline 0300362600 & 19 & NGC 5316 & $11.6556_{-0.0075}^{+0.0007}$ & $1602.169_{-0.004}^{+0.003}$ & $0.213_{-0.005}^{+0.005}$ & $0.20_{-0.14}^{+0.18}$ & $13.7_{-1.4}^{+1.5}$ & $0.25_{-0.07}^{+0.09}$ & $0.15_{-0.05}^{+0.05}$ & $0.19_{-0.05}^{+0.05}$ & $89.2_{-0.8}^{+0.6}$ & $4.6_{-0.5}^{+0.5}$ & $51.3_{-5.2}^{+6.0}$ & \\
\hline 0306385801 & 20 & NGC 3532 & $14.0367_{-0.0018}^{+0.0002}$ & $5.028_{-0.003}^{+0.003}$ & $0.110_{-0.002}^{+0.003}$ & $0.48_{-0.30}^{+0.19}$ & $15.2_{-2.3}^{+1.8}$ & $0.24_{-0.09}^{+0.10}$ & $0.17_{-0.05}^{+0.05}$ & $0.21_{-0.05}^{+0.05}$ & $86.9_{-2.0}^{+1.6}$ & $2.3_{-0.3}^{+0.4}$ & $25.5_{-2.8}^{+4.5}$ & \\
\hline 0308538095 & 21 & NGC 2516 & $11.6929_{-0.0002}^{+0.0002}$ & $1420.294_{-0.002}^{+0.0002}$ & $0.130_{-0.003}^{+0.004}$ & $0.40_{-0.27}^{+0.24}$ & $18.6_{-1.0}^{+0.9}$ & $0.63_{-0.10}^{+0.10}$ & $0.28_{-0.05}^{+0.05}$ & $0.29_{-0.05}^{+0.05}$ & $84.6_{-2.0}^{+3.2}$ & $1.6_{-0.1}^{+0.1}$ & $18.2_{-1.0}^{+1.0}$ & \\
\hline 0317536999 & 22 & Ruprecht 94 & $5.8557_{-0.0007}^{+0.0007}$ & $1576.007_{-0.004}^{+0.004}$ & $0.178_{-0.025}^{+0.037}$ & $0.90_{-0.08}^{+0.06}$ & $10.4_{-0.8}^{+0.7}$ & $0.44_{-0.09}^{+0.09}$ & $0.15_{-0.05}^{+0.05}$ & $0.17_{-0.05}^{+0.05}$ & $84.5_{-1.5}^{+0.8}$ & $3.2_{-0.5}^{+0.7}$ & $36.1_{-5.7}^{+7.9}$ & \\
\hline 0372913337 & 23 & NGC 2516 & $1.7860_{-0.0001}^{+0.0001}$ & $1325.341_{-0.006}^{+0.006}$ & $0.045_{-0.004}^{+0.011}$ & $0.96_{-0.10}^{+0.03}$ & $3.7_{-0.5}^{+0.5}$ & $0.22_{-0.07}^{+0.09}$ & $0.15_{-0.05}^{+0.05}$ & $0.19_{-0.05}^{+0.05}$ & $71.1_{-16.1}^{+4.9}$ & $1.1_{-0.2}^{+0.3}$ & $11.8_{-2.4}^{+3.5}$ & \\
\hline 0389927567 & 24 & Melotte 101 & $6.9408_{-0.0010}^{+0.0009}$ & $1575.805_{-0.006}^{+0.006}$ & $0.261_{-0.036}^{+0.058}$ & $0.85_{-0.14}^{+0.10}$ & $10.0_{-1.3}^{+1.1}$ & $0.28_{-0.09}^{+0.10}$ & $0.15_{-0.05}^{+0.05}$ & $0.17_{-0.05}^{+0.05}$ & $80.1_{-5.0}^{+2.7}$ & $5.7_{-1.0}^{+1.3}$ & $64.1_{-11.0}^{+14.6}$ & \\
\hline 0410450228 & 25 & NGC 2516 & $15.7784_{-0.0004}^{+0.0004}$ & $1420.250_{-0.004}^{+0.0004}$ & $0.095_{-0.010}^{+0.022}$ & $0.93_{-0.04}^{+0.04}$ & $21.0_{-1.5}^{+1.2}$ & $0.50_{-0.10}^{+0.09}$ & $0.22_{-0.05}^{+0.05}$ & $0.25_{-0.05}^{+0.05}$ & $87.1_{-0.9}^{+0.4}$ & $\begin{array}{l}1.4_{-0.2}^{+0.3} \\
\text { (1) }\end{array}$ & $15.7_{-2.0}^{+3.7}$ & TOI-681 \\
\hline 0413809436 & 26 & NGC 5925 & $0_{-0.0006}^{+0.0006}$ & $.336_{-0.003}^{+0.003}$ & $0.233_{-0.007}^{+0.011}$ & $0.52_{-0.31}^{+0.18}$ & $6.4_{-0.5}^{+0.4}$ & $0.49_{-0.10}^{+0.10}$ & $0.18_{-0.05}^{+0.05}$ & $0.23_{-0.05}^{+0.05}$ & $84.3_{-1.9}^{+2.8}$ & $3.6_{-0.3}^{+0.4}$ & $40.2_{-2.9}^{+4.4}$ & \\
\hline 0419091401 & 27 & Ruprecht 48 & $12.7071_{-0.0023}^{+0.0002}$ & $1499.685_{-0.007}^{+0.007}$ & $0.188_{-0.011}^{+0.047}$ & $0.75_{-0.28}^{+0.16}$ & $11.9_{-2.2}^{+2.0}$ & $0.14_{-0.06}^{+0.08}$ & $0.16_{-0.09}^{+0.00}$ & $0.21_{-0.10}^{+0.10}$ & $81.4_{-7.6}^{+3.3}$ & $5.2_{-0.9}^{+1.5}$ & $57.9_{-10.6}^{+17.2}$ & \\
\hline 0432564189 & 28 & Ruprecht 82 & $3.7611_{-0.0003}^{+0.00003}$ & $1546.783_{-0.004}^{+0.0004}$ & $0.120_{-0.005}^{+0.008}$ & $\begin{array}{r}0.64_{-0.42}^{+0.20} \\
0.00\end{array}$ & $6.5_{-0.8}^{+0.7}$ & $0.27_{-0.09}^{+0.09}$ & $0.16_{-0.05}^{+0.05}$ & $0.21_{-0.05}^{-0.05}$ & $76.1_{-9.3}^{+5.6}$ & $2.4_{-0.3}^{+0.4}$ & $\begin{array}{r}-1.0 \\
27.3_{-3.1}^{+5.0}\end{array}$ & \\
\hline 0450610413 & 29 & Harvard 5 & $10.6780_{-0.0027}^{+0.0027}$ & $1600.682_{-0.004}^{+0.004}$ & $0.176_{-0.004}^{+0.004}$ & $0.21_{-0.15}^{+0.19}$ & $15.3_{-1.2}^{+1.0}$ & $0.42_{-0.09}^{+0.10}$ & $0.15_{-0.05}^{+0.05}$ & $0.20_{-0.05}^{+0.05}$ & $89.2_{-0.7}^{+0.6}$ & $3.0_{-0.2}^{+0.3}$ & $33.9_{-2.5}^{+2.9}$ & \\
\hline 0460205581 & 30 & IC 2602 & $8.3252_{-0.0006}^{+0.0006}$ & $1574.271_{-0.002}^{+0.002}$ & $0.059_{-0.002}^{+0.005}$ & $0.57_{-0.35}^{+0.26}$ & $16.9_{-0.6}^{+0.6}$ & $0.94_{-0.10}^{+0.10}$ & $0.31_{-0.05}^{+0.05}$ & $0.30_{-0.05}^{+0.05}$ & $83.4_{-2.4}^{+2.7}$ & $0.6_{-0.1}^{+0.1}$ & $6.9_{-0.4}^{+0.6}$ & TOI- 837 \\
\hline 0460950389 & 31 & IC 2602 & $2.8622_{-0.0004}^{+0.0004}$ & $1572.224_{-0.004}^{+0.004}$ & $0.042_{-0.002}^{+0.002}$ & $0.30_{-0.20}^{+0.26}$ & $9.4_{-0.2}^{+0.2}$ & $1.37_{-0.10}^{+0.10}$ & $0.43_{-0.05}^{+0.05}$ & $0.38_{-0.05}^{+0.05}$ & $88.1_{-1.3}^{+1.3}$ & $0.3_{-0.1}^{+0.1}$ & $3.8_{-0.2}^{+0.2}$ & \\
\hline 0462004618 & 32 & NGC 3114 & $2.3669_{-0.0001}^{+0.0001}$ & $1544.599_{-0.004}^{+0.004}$ & $0.172_{-0.024}^{+0.027}$ & $0.93_{-0.05}^{+0.04}$ & $5.1_{-0.5}^{+0.4}$ & $0.32_{-0.09}^{+0.08}$ & $0.15_{-0.05}^{+0.05}$ & $0.20_{-0.05}^{+0.05}$ & $78.2_{-3.9}^{+1.9}$ & $3.3_{-0.5}^{+0.6}$ & $37.3_{-5.9}^{+6.6}$ & \\
\hline 0748919024 & 33 & Ruprecht 151 & $2.5395_{-0.0006}^{+0.0007}$ & $1494.064_{-0.004}^{+0.004}$ & $0.118_{-0.003}^{+0.004}$ & $0.37_{-0.25}^{+0.26}$ & $6.0_{-0.5}^{+0.4}$ & $0.46_{-0.11}^{+0.10}$ & $0.25_{-0.05}^{+0.05}$ & $0.27_{-0.05}^{-0.05}$ & $86.9_{-1.7}^{+2.1}$ & $1.7_{-0.1}^{+0.2}$ & $19.2_{-1.4}^{+1.9}$ & \\
\hline 1036769612 & 34 & Trumpler 22 & $7.4732_{-0.0013}^{+0.0014}$ & $1599.298_{-0.005}^{+0.005}$ & $0.111_{-0.004}^{+0.005}$ & $0.37_{-0.25}^{+0.24}$ & $13.4_{-1.6}^{+1.3}$ & $0.57_{-0.19}^{+0.19}$ & $0.24_{-0.05}^{+0.05}$ & $0.27_{-0.05}^{+0.05}$ & $88.1_{-1.2}^{+1.2}$ & $1.5_{-0.2}^{+0.2}$ & $17.0_{-1.7}^{+2.6}$ & \\
\hline
\end{tabular}

